\title{
A novel non-metabolic function of lactate in remodeling uterine receptivity by inducing endometrial histone lactylation, revealed by a proteomic atlas of maternal-fetal crosstalk in sheep
}

\section{Qianying Yang}

China Agricultural University https://orcid.org/0000-0003-0077-7437

Juan Liu

China Agricultural University

Yue Wang

China Agricultural University

Wei Zhao

China Agricultural University

\section{Wenjing Wang}

China Agricultural University

Jian Cui

China Agricultural University

Jiajun Yang

China Agricultural University

\section{Yuan Yue}

China Agricultural University

\section{Meiqiang Chu}

China Agricultural University

Qingji Lv

China Agricultural University

\section{Lizhu Ma}

China Agricultural University

\section{Yawen Tang}

China Agricultural University

\section{Yupei Hu}

China Agricultural University

\section{Kai Miao}

University of Macau https://orcid.org/0000-0003-4832-3354

Haichao Zhao

China Agricultural University 


\section{Jianhui Tian}

China Agricultural University

Lei An ( $\square$ anleim@cau.edu.cn )

China Agricultural University

\section{Article}

Keywords: lactate, uterine receptivity, endometrial histone lactylation, proteomic atlas

Posted Date: May 6th, 2021

DOl: https://doi.org/10.21203/rs.3.rs-299619/v1

License: (c) (1) This work is licensed under a Creative Commons Attribution 4.0 International License. Read Full License 


\section{Abstract}

Well-orchestrated maternal-fetal crosstalk involves secreted ligands, interacting receptors, and coupled pathways between the conceptus and endometrium. However, previous researches mainly focused on either the conceptus or endometrium in isolation. The lack of integrated analysis, especially on protein levels, has made it challenging to advance our understanding of the crosstalk. Herein, focusing on ligand-receptor complexes and coupled pathways at maternal-fetal interface in sheep, a well-established embryo implantation model, we provide the first comprehensive proteomic atlas of ligand-receptorpathway cascades that are essential for implantation. Based on these candidate interactions, we further revealed the physical interaction of albumin-claudin 4 and their role in facilitating embryo attachment to endometrium. More interestingly, we demonstrated a novel non-metabolic function of enhanced conceptus glycolysis in remodeling uterine receptivity, by inducing endometrial histone lactylation, a newly identified histone modification. Our results from in vitro and in vivo models supported the essential role of lactate, as a key pregnancy recognition signal, in regulating redox homeostasis and apoptotic balance to ensure successful implantation. Our study identified many putative molecular and cellular mechanisms that fine-tuned conceptus-endometrium crosstalk during implantation, thus providing important clues for developing potential clinical intervention strategies to improve pregnancy outcomes following both natural conception and assisted reproduction.

\section{Introduction}

In mammals, successful implantation and healthy pregnancy depend on well-orchestrated crosstalk between the developmentally competent conceptus and the receptive endometrium, [1-3]. Early pregnancy loss occurring during the peri-implantation period is a relatively common phenomenon in humans and other low-ovulating mammalian species. In natural conception, approximately $75 \%$ of failed pregnancies are considered to be caused by implantation failure $[1,4,5]$. Failed implantation is also a major limiting factor in assisted reproduction [6]. Therefore, a comprehensive understanding of the wellorchestrated conceptus-endometrium crosstalk at the implantation stage is of importance to improve the outcomes of natural pregnancy and pregnancy achieved via in vitro fertilization (IVF).

Although current molecular and cellular mechanisms that govern conceptus-endometrium crosstalk have been gleaned primarily from mouse models $[1,3,7]$, ruminant models have contributed key insights and updated our knowledge of conceptus-endometrium interactions. Compared with the short length of the implantation window in murine models, the prolonged period of apposition and attachment in cows and sheep makes them outstanding candidate models to study the human conceptus-endometrium interactions $[8,9]$. The role of the immunosuppressive interferon $\tau$ (IFNT), derived from the trophectoderm, in the maternal recognition of pregnancy was initially shown in sheep [10]. It has been well-established that IFNT acts locally on the endometrium to prevent luteolysis by inhibiting the synthesis and release of prostaglandin $\mathrm{F} 2 \mathrm{a}(\mathrm{PGF} 2 \mathrm{a})$, thus allowing continued production of progesterone by the functional corpus luteum to establish and maintain pregnancy [11-13]. Subsequent studies further reported that IFNT also fine-tunes a range of other physiological processes in endometrial remolding by stimulating or 
suppressing the expression of certain genes [14-17]. IFNT provides a classic illustration of how the establishment of uterine receptivity depends on a conceptus-originated biochemical signal. Other paracrine signals, such as cortisol, secreted phosphoprotein 1, and prostaglandins, which contribute to successful implantation and the maintenance of pregnancy, were also deciphered using ruminant models $[12,18,19]$. In addition, using bovine embryos of different origins, it was shown that the endometrium responds differently to embryos with different developmental potential, and the limits of endometrial plasticity provided new insights into the contribution of embryo-maternal interactions to successful implantation [20,21]. More recently, paired conceptus-endometrium transcriptome analyses of individual pregnancies in bovine provided the first evidence that the conceptus and endometrium are fine-tuned and coordinated at the level of an individual pregnancy [22].

Fine-tuned and reciprocal crosstalk involves secreted ligands, their interacting receptors, and coupled pathways between the conceptus and endometrium. Until now, most research has focused on either the conceptus [23-26] or the endometrium [20,21,27-29] in isolation, the lack of integrated analysis between the paired conceptus and endometrium has made it challenging to advance our understanding of the pathways and functions that govern conceptus-endometrium crosstalk during implantation. More recently, disrupted crosstalk following somatic cell nuclear transfer (SCNT) [30], IVF [31], and fertilityclassified animals [32] have been profiled, and revealed important clues to failed implantation and pathological pregnancy induced by impaired conceptus-endometrium crosstalk. Despite this, the functional interactions in normal pregnancies remain to be explored in depth. Recently, a repository of ligand-receptor complexes based on high-throughput data provided a statistical tool to predict the cellcell communications of diverse physiological and pathological processes, through evaluating their molecular interactions [33-35]. Despite the growing evidence of conceptus-endometrium interactions at the gene expression level, the communications on the protein level, which can provide more direct and accurate understanding, have never been characterized.

In the present study, using sheep as the model, we present a comprehensive proteomic atlas of crosstalk at maternal-fetal interface, and thus reconstructing a repository of the potential ligand-receptor-pathway cascade. In addition, by highlighting the metabolic shift in the conceptus, we also proposed the potential role of lactate-induced histone lactylation, a newly identified histone modification, in remodeling endometrial receptivity.

\section{Results}

\section{Identifying differentially abundant membrane and secreted proteins between the conceptus and endometrium.}

To profile the proteome in the conceptus and endometrium by implantation stage, we collected conceptuses, endometrial caruncular (C), and intercaruncular (IC) areas (Fig. 1A) from thirty-six pregnant sheep on day 17 of pregnancy, which is the time of filamentous conceptus attachment in the sheep uterus $[36,37]$ that is frequently selected to explore the mechanisms of conceptus-endometrium crosstalk 
$[31,38,39]$. Maternal-fetal crosstalk in ruminants is characterized by interaction occurring in both the $C$ and IC areas. Aglandular $\mathrm{C}$ areas serve as the sites of superficial attachment and placentation, whereas glandular IC areas, which are rich in branched and coiled uterine glands, are mainly responsible for the synthesis and secretion of histotroph $[12,40]$. We divided the thirty-six samples into equal three pools (12 samples/pool) as biological replicates, and sequenced their proteomes, which showed high reproducibility (Fig. S1A). Finally, we identified 1468, 1494, and 1575 proteins in the conceptus, C area, IC area, respectively (Fig. 1A and Table S1). When hierarchical classification was applied, the endometrial C and IC areas clustered closely together, whereas the conceptus samples were categorized separately (Fig. S1B), which was also recapitulated by the results of principal component analysis (PCA) (Fig. 1B). Comparative analysis of differentially abundant proteins (DAPs) (false discovery rate (FDR) $<0.05$, fold change $(\mathrm{FC})>2$ ) identified 196 and 232 proteins that were significantly more abundant in the conceptus compared with those in the $\mathrm{C}$ or IC areas; whereas 224 and 325 proteins were significantly more abundant in the $\mathrm{C}$ and IC areas compared with those in the conceptus, and a substantial proportion of DAPs were specifically enriched in endometrial or conceptus tissues (Figs. S1C and S1D).

To explore the important interactions between the conceptus and endometrium systematically, we screened out differentially abundant membrane and secreted proteins based on subcellular localization annotations from UniProt (Fig. 1C). Secreted proteins appeared to be more enriched in the C or IC areas than in the conceptus, which supported the previous notion that the endometrium is an active site of cytokine production and action [41]. Next, we used 304 common DAPs between the conceptus and endometrial tissues to construct a protein-protein network, and found nine high-scoring proteins (interaction edges $>40$ ), including the secreted protein fibronectin (FN1) and the membrane protein $40 \mathrm{~S}$ ribosomal protein SA (RPSA), both of which are essential adhesion molecules (Fig. S1E). To investigate the conceptus-endometrium crosstalk deeply, we next focused on the following three aspects: Differentially abundant membrane proteins, differentially abundant secreted proteins, and differentially enriched pathways.

\section{Screening differentially abundant membrane proteins and their interacting secreted partners.}

Having identified differentially abundant membrane and secreted proteins of the maternal-fetal interface, we next attempted to screen potential interacting partners that might be essential for conceptusendometrium crosstalk during implantation. To this end, we first focused on the membrane proteins that changed commonly between the conceptus and endometrial tissues (FDR $<0.05, F C>2)$. We screened out their interacting secreted partners based on the interaction score from STRING and the protein subcellular location from UniProt (Table S2). Many of the high-scoring interactions involve membrane transporter proteins (solute carrier family 2 member 1 (SLC2A1), calcineurin like EF-hand protein 1 (CHP1), glutamicoxaloacetic transaminase 2 (GOT2)), heat-shock protein family members (heat-shock protein 90 alpha family class B member 1 (HSP90AB1), heat shock protein family A member 8 (HSPA8)), and cell adhesion molecules (claudin 4 (CLDN4), basal cell adhesion molecule (BCAM)) (Fig. 1D). Phenotype annotations suggested that these membrane proteins might be essential for embryonic and fetal development and survival (Fig. 1D). In addition to differentially abundant membrane proteins common to 
endometrial tissues relative to the conceptus, we also examined differentially abundant membrane proteins ( $F D R<0.05, F C>2$ ) specific to the $\mathrm{C}$ or IC areas, and their interacting secreted partners (Figs. S2A and S2B), which might provide further candidates to investigate the different roles of the $C$ and IC areas in supporting conceptus implantation.

To further validate the important role of the predicted membrane partners in supporting conceptusendometrium crosstalk, we next characterized the expression patterns of these candidates in the endometria of successful and failed pregnancies using our previously published proteomic data [42]. We found that the levels of a majority of these candidates changed significantly $(P<0.05)$ in the endometrium that underwent pregnancy failure, implying the essential functions of these membrane partners in supporting a successful pregnancy (Fig. S2C). Interestingly, we noticed that protein tyrosine kinase 7 (PTK7), an evolutionarily conserved transmembrane receptor, was significantly more abundant in the endometrium that underwent pregnancy failure compared with that in the successful pregnancy (Fig. S2C), and its mRNA expression level was also significantly reduced in the human endometrium from the pre-receptive to receptive phase (Fig. S2D), suggesting that a fine-tuned PTK7 level is essential for the normal physiological functions of the endometrium. Meanwhile, we also found that the levels of many of our screened membrane partners changed significantly $(P<0.05)$ in endometrial tissues of patients with endometriosis compared with those in the healthy endometrium (Fig. S2E), suggesting that these interacting membrane partners might also participate in uterine pathology.

\section{Screening differentially abundant secreted proteins and their interacting membrane partners.}

Having screened potential interacting partners based on differentially abundant membrane proteins, we next focused on differentially abundant secreted proteins (FDR $<0.05, F C>2)$ and their interacting membrane partners (Fig. 1E and Figs. S3A and S3B). Many of the high-scoring interactions involved cell adhesion molecules (FN1, collagen type $\mathrm{VI}$ alpha 1 chain (COL6A1), collagen type $\mathrm{VI}$ alpha 2 chain (COL6A2)), glycoprotein family members (alpha 2-HS glycoprotein (AHSG), alpha-1-B glycoprotein (A1BG)), and complement components (complement C5 (C5), complement C9 (C9), plasminogen (PLG)). The MGI phenotype annotations suggested that these membrane partners might be essential for embryonic and fetal development and survival (Fig. 1E). Given that more screened secreted proteins derived from the endometrium than those from the conceptus, our results of paired interactions also support the notion that endometrial tissues might be more active in supporting the crosstalk at the maternal-fetal interface. Similarly, reanalysis of secreted partners using previously published proteomic or transcriptomic data also supported the physiological or pathological significance of our screened candidates (Figs. S3C-E).

\section{Construction of a repository of the potential ligand-receptor- pathway cascade}

Having characterized conceptus-endometrium crosstalk using interacting secreted and membrane partners, we next attempted to further understand the critical pathways and biological processes that 
may couple to ligand-receptor complexes to support the crosstalk at the maternal-fetal interface. Therefore, functional profiling was performed using proteins that were more abundant in endometrial or conceptus tissues (Figs. S4A-D). We found that proteins that were abundant in endometrial tissues were functionally associated with glutathione metabolism, complement and coagulation cascades, and focal adhesion (Fig. S4A), as well as biological processes of cell adhesion, response to virus, and actin crosslink formation (Fig. S4B). By contrast, using proteins that were abundant in the conceptus, we identified that pathways or biological processes of energy metabolism, cellular oxidant detoxification, cell-cell adhesion, and protein stabilization were significantly enriched (Figs. S4C and S4D). Based on the analyses of differentially abundant secreted proteins, membrane proteins, and enriched pathways between the conceptus and C or IC areas, we integrated them to reconstruct a repository of the potential ligand-receptor-pathway cascade, which includes bidirectional cascades: Secreted proteins from the $\mathrm{C}$ or IC areas act on membrane partners on the conceptus and in turn promote the development of the conceptus; Secreted proteins of the conceptus act on membrane partners of the $\mathrm{C}$ or IC areas and in turn promote endometrial remodeling. Among these, some of candidate cascades have been identified, such as laminins-integrin-cell adhesion and collagens-integrin-cell adhesion [43]; whereas many cascades have not been identified at embryo-maternal interface, such as ALB-CLDN4-cell adhesion and fibronectin 1 (FN1)- protein tyrosine kinase 7 (PTK7)-cell invasion (Fig. 2A). Particularly, the albumin is one of the most abundant proteins in uterine luminal fluid $[44,45]$ and was reported to support embryo development by carrying energy sources, osmoregulators, pH stabilizers, or scavenging ions and toxins [46], but its role in mediating conceptus-endometrium crosstalk has never been determined. We therefore focused our analyses on ALB-CLDN4-cell adhesion cascade. Our results showed that $5 \mu \mathrm{g} / \mathrm{mL}$ albumin exposure not only significantly enhanced the adhesiveness of Ishikawa cells (Fig. S5A), but also significantly improved the attachment of blastocysts (Fig. 2B). Moreover, the short-term (30 min) protein overload assay showed a significant increase in ALB binding interactions on the cell surface of blastocysts exposed to mouse serum albumin relative to control (Fig. S5B). More importantly, this increased binding interactions was also verified using the in vitro implantation model (Fig. $2 \mathrm{C}$ and Fig. S5C), and the Co-immunoprecipitation (Co-IP) of CLDN4 provided direct evidences of the physical interaction between ALB and CLDN4 (Fig. 2D), as predicted by the protein-protein docking model (Fig. S5D). Furthermore, in vivo results from E6.5 mouse embryo-maternal interface showed mutually exclusive expression patterns of $\mathrm{Alb}$ and $\mathrm{Cldn} 4$ during implantation (Fig. 2E), which was similar to those in day 17 sheep conceptus and C or IC areas (Fig. 1D). Notably, in situ immunostaining and Co-IP of E6.5 showed an obvious co-localization and direct interaction of ALB and CLDN4 at embryo-maternal interface (Figs. 2F and 2G; Fig. S5E). Collectively, we proved, in vitro and in vivo, the important role of ALB-CLDN4-cell adhesion cascade in embryo-maternal crosstalk during implantation.

\section{The key pathways and processes at the maternal-fetal interface.}

To further investigate the conceptus-endometrium crosstalk deeply, we constructed and clustered a pathway network using PathwayConnector (PC) [47] to find key nodes functionally linked with an endometrial receptive status. Three sub-clusters and their key pathways were noteworthy: Sub-cluster 1 : Complement and coagulation cascades and apoptosis; sub-cluster 2: Phosphatidylinositol-3-kinase 
(PI3K)-protein kinase B (AKT) and mitogen activated protein kinase (MAPK) signaling pathway; subcluster 3: Glutathione metabolism (Fig. 3A). Similarly, we constructed the direct connections using the enriched pathways in the conceptus. Two sub-clusters and their key pathways were clustered: Sub-cluster 1: Glycolysis/gluconeogenesis; Sub-cluster 2: The PI3K-AKT signaling pathway (Fig. 3B). Furthermore, the physiological significance of these enriched pathways for conceptus-endometrium crosstalk during implantation was further supported via Gene Set Enrichment Analysis (GSEA) of the conceptus and endometrial transcriptomes on day 12 and 16 of pregnancy [45]. Our results of reanalysis indicated that glycolysis/gluconeogenesis (normalized enrichment score $(\mathrm{NES})=1.35$, FDR q-value $=0.068$ ) is enriched in the conceptus on day 16 of pregnancy (Fig. 3C), while apoptosis, glutathione metabolism, and complement and coagulation cascades are enriched in the endometrium on day 16 of pregnancy (Fig. 3C).

\section{The receptive response of the endometrium to the embryonic lactate signal.}

Based on the observation of enriched glycolysis/gluconeogenesis in the conceptus at the maternal-fetal interface, we next profiled gene expression dynamics of glycolysis-related enzymes in the conceptus from day 12 to 20 of pregnancy (Fig. 3D) using previously published transcriptomic data [45]. The results showed a tendency towards enhanced glycolysis, which agreed with that reported in other species [4850]. Given that glycolysis is less efficient in terms of ATP production compared with oxidative phosphorylation, it has been thought that there might be non-energy providing functions of glycolysis in peri-implantation embryos [51, 52]. This was reminiscent of the functions of lactate, the metabolic byproduct of glycolysis. It has been reported that lactate production increases significantly at implantation in mice and humans [53-55]. In line with this, we detected relatively high levels of lactate in the conceptus and endometrial tissues (Fig. 3E), which were comparable to those in tumor cells [56]. It should be also emphasized that in the endometrium undergoing pregnancy failure, lactate concentrations were much lower in both the $\mathrm{C}$ and IC areas compared with that in their counterparts in the successful pregnancy (Fig. 3E). This fact, together with the results that an important lactate-preferring transporter (monocarboxylate transporter, MCT1) was upregulated in the endometrium during implantation (Fig. S5F), suggested that lactate might play a critical role in manipulating the microenvironment for uterine implantation. Interestingly, we noticed that the chaperone glycoproteins, immunoglobulin family, which interact with monocarboxylate transporters (MCTs) to maintain their stability [57], showed tissue-biased expression patterns (Fig. S5F), indicating that MCT1 in the conceptus might interact preferentially with basigin (BSG), whereas MCT1 in the endometrium might interact preferentially with embigin (EMB).

Notably, a recent study showed that lactate-derived lactylation of histone residues is an important epigenetic modification that directly regulates gene transcription [58]. Therefore, we hypothesized that increased lactate levels at the maternal-fetal interface might serve as the donor to stimulate histone lactylation and thus facilitate remodeling processes to prepare a receptive endometrium. To test this, we first detected the H3K18la level, a subtype of recently identified histone lactylation that can respond to increased lactate concentration in mouse and human cells [58], in the $\mathrm{C}$ and IC areas using western blotting. We detected high levels of H3K18la in the C and IC areas on day 17 of pregnancy. By contrast, 
H3K18la levels were significantly lower in the endometrium undergoing pregnancy failure, implying the important role of H3K18la in establishing endometrial receptivity (Fig. 3F). Next, we used a wellestablished in vitro implantation model based on mouse embryo attachment to Ishikawa cells (a human endometrial cell line) $[59,60]$ to test if histone lactylation in the endometrium was associated with embryo-originated stimulations. We found H3K18la levels of Ishikawa cells showed a declining tendency from the proximal to the distal fields (Figs. $3 \mathrm{G}$ and S5G), which suggested that embryos could stimulate H3K18la in the endometrium. In addition, exogenous supplementation of sodium L-lactate recapitulating embryo-originated lactate, induced a significant increase in H3K18la levels in endometrial cells (Fig. $3 \mathrm{H})$. These facts also suggested that lactate induced H3K18la in the endometrium appears to be common across species

\section{Glutathione (GSH)-based cell redox homeostasis is the target of lactate-induced histone lactylation.}

Next, we attempted to study the potential physiological role of lactate-induced lactylation in endometrial remolding during implantation. We focused on the potential H3K18la-associated endometrial genes and pathways via a Venn diagram consisting of the following data: (1) 1784 genes whose promoters were enriched with H3K18la (GSE115354), as the putative H3K18la-regulated targets; (2) 2077 genes whose expression changed significantly in the sheep luminal epithelium from day 12 to 16 of pregnancy (GSE87017), and (3) 2228 genes whose expression changed significantly in the sheep glandular epithelium from day 12 to 16 of pregnancy (GSE87017), as the potential candidates responsible for endometrial remodeling. This analysis identified 44 candidates that might be regulated by H3K18la in the endometrium during embryo implantation (Fig. S6A). Functional annotations showed that these genes are involved in the biological processes of cell redox homeostasis, apoptosis, cytoskeleton, proteasome, cell proliferation, cell migration, cell adhesion, and immune response (Fig. S6A). In addition, the significant responses of these processes to lactate were further validated by GSEA of previously published transcriptome data (GSE115354): Apoptosis, cell adhesion molecules, negative regulation of immune response, the PI3K-AKT signaling pathway, and positive regulation of cell proliferation were significantly enriched in lactate treated mouse cells (NES <-1, FDR q-value < 0.25) (Fig. S6B). Among these, glutathione (GSH)-mediated cell redox homeostasis, as well as related pathways, e.g., reactive oxygen species (ROS) positive response and apoptosis, attracted our attention. ROS at a controlled level has been reported to serve as the critical mediator of endometrial remodeling [61,62]. This was also supported by our observation that ROS positive response genes [63] were enriched significantly in the day 16 endometrium relative to that in the day 12 endometrium, and in the endometrium on day 17 of pregnancy relative to that in pregnancy failure (NES > 1, FDR q-value < 0.25) (Figs. S6C and S6D); while apoptosis, which is conducive to trophoblast invasion at the site of embryo attachment [64], was also upregulated in sheep endometrium during implantation (Fig. 3C). Thus, we next used Ishikawa cells, which can respond to lactate-induced lactylation, as the model to test if lactate could induce ROS production and apoptosis in the endometrium. As expected, sodium L-lactate supplementation in the culture medium increased ROS levels and apoptotic rate significantly in a dose-dependent manner (Figs. 4A and 4B). 
The GSH-based antioxidative system is functionally active at the maternal-fetal interface and is critical for the establishment of pregnancy $[65,66]$. In line with this, many genes involved in GSH metabolism and function was upregulated in the sheep endometrium during the implantation stage (Fig. S6E), which was consistent with the result of GSEA in Fig. 3C. Correspondingly, the importance of the GSH-based antioxidative system in successful implantation was also supported by the analyses of GSEA and differentially abundant proteins related to glutathione metabolism of the endometrium in successful and failed pregnancies (Figs. S6D and S6F). These results, together with clues suggesting that the GSH-based antioxidative system might be the target of lactate-induced H3K18la in the endometrium during implantation, led us to test whether lactate could stimulate GSH metabolism and function. Interestingly, we found that a relative low-level $(10 \mathrm{mM})$, but not a high-level $(50 \mathrm{mM}, 100 \mathrm{mM})$ of sodium L-lactate supplementation significantly promoted the ratio of GSH/GSSG (Fig. 4C), implying enhanced intracellular antioxidative activity $[67,68]$, while excessive ROS production induced by high-level lactate (Fig. 4A) might consume redundant GSH.

Finally, we attempted to determine if lactate production in the uterine microenvironment is beneficial for implantation. To this end, we first detected the proliferation of Ishikawa cells, because uterine cell proliferation is the prerequisite for establishing endometrial receptivity [7]. We found that $10 \mathrm{mM}$ sodium L-lactate stimulated significant and sustained proliferation of Ishikawa cells (Fig. 4D). More importantly, the in vitro mouse embryo attachment assay indicated that sodium L-lactate supplementation could improve embryo attachment in a time- and dose-dependent manner: $10 \mathrm{mM}$ sodium L-lactate supplementation led to a significant increase in the attachment ratio, whereas excessive or prolonged lactate exposure resulted in unexpected embryonic degradation and endometrial cell death (Fig. 4E and Fig. S6G), implying that well-controlled lactate production acts as a pregnancy recognition signaling molecule. These findings were further verified in vivo. Intrauterine injection of lactate dehydrogenase inhibitor (oxamate) on day 4 of pregnancy significantly reduced implantation sites on day 5 in a dosedependent manner. Notably, this implantation defects, could be rescued by exogenous addition of the relative low-level ( $0.04 \mathrm{mM}$ and $0.2 \mathrm{mM})$, but not high-level lactate ( $1 \mathrm{mM}$ and $5 \mathrm{mM}$ ) (Figs. $4 \mathrm{~F}$ and $4 \mathrm{G}$ ). Collectively, we suggested that a fine-tuned lactate production is essential and beneficial for embryo implantation.

\section{Discussion}

Despite the frequently reported high-throughput analyses that have been used to characterize the transcriptomic and proteomic features of the embryo/conceptus and endometrium at the implantation stage in mice [23,69], humans [35, 70,71], and domestic animals [22, 72-75], few studies have focused on the crosstalk at the maternal-fetal interface. Using sheep as the model, we provided a comprehensive proteomic atlas of conceptus-endometrium crosstalk at the implantation stage in this ruminant species. Particularly, our study presents the ligand-receptor interaction landscape at the maternal-fetal interface. By highlighting the metabolic shift in the conceptus, we also proposed the potential role of lactateinduced lactylation in remodeling endometrial receptivity. 
Based on the well-established database of membrane and secreted proteins, we predicted a variety of ligand-receptor complexes at the protein level that might be essential for conceptus-endometrium crosstalk. The predictability of our constructed interaction map was supported by a series of membrane or secreted partners that have been identified as key regulators of successful implantation or uterine pathology, such as calpain 2 (CAPN2) [76], integrin subunit alpha 9 (ITGA9) [43, 77], and non-metastatic gene 23-H1 (NME1) [78, 79]. For example, membrane protein CAPN2 is highly expressed in endometrial tissues, while its interacting secreted protein cathepsin B (CTSB) is enriched in the conceptus (Fig. 1D). CAPN2 is concentrated along the basal cell surface of rat uterine luminal epithelial cells at the time of implantation, and plays a key role in focal adhesion disassembly and uterine receptivity [76], while CTSB has been reported to play a critical role in endometrial remodeling $[80,81]$. Our study also revealed the interactions between secreted and membrane adhesion molecules. We found that the expression of FN1 gradually increases in the conceptus from day 12 to 20 of pregnancy and is primarily enriched and secreted by the conceptus (Fig. $\mathbf{S 6 H}$ ); whereas its high-scoring interacting membrane partner, integrin subunit beta 1 (ITGB1), a glycoprotein that is localized on the epithelial membrane [82, 83], is more abundant in endometrial tissues at the maternal-fetal interface. The integrin-mediated FN1 binding activity of trophoblast cells has been identified to strengthen trophoblast adhesion to the endometrial extracellular matrix [84, 85].

Our results also indicated candidate interactions associated with both implantation and uterine diseases: Membrane protein NME1, a wide-spectrum tumor metastasis suppressor, and its interacting secreted partner, FK506 binding protein 1A (FKBP1A), a member of the immunophilin protein family, are enriched in the conceptus and endometrial tissues respectively (Fig. 1E). Our reanalysis showed that the level of NME1 was significantly lower in endometrium of endometriosis (Fig. S2E), which is in line with the previous notion that NME1 plays an important role in regulating the invasiveness of trophoblast cells and participates in the pathogenesis of endometriosis [78, 79]. Similarly, the level of FKBP1A is also significantly lower in endometrium of endometriosis (Fig. S3E), which agreed with the report that FKBP1A is upregulated in the endometrium of blocked or reduced the number of endometriotic vesicles [86].

More importantly, we also identified a variety of putative interactions that have not been shown previously to participate in crosstalk at the maternal-fetal interface, including conceptus-secreted and endometrial membrane partners (CD109-ALPL, PRXL2A-HSP90AB1, and FN1-PTK7), as well as endometrium-secreted and conceptus-enriched partners (ALB-CLDN4, DCN-AGRN, and GC-LRP2).

Collectively, based on the well-established and potential receptor-ligand complexes, we constructed a map of conceptus-endometrium crosstalk during implantation (Fig. 2A). On the one hand, conceptussecreted proteins could interact with their endometrial membrane partners and remodel the endometrial receptivity by regulating the biological processes of cell adhesion, complement and coagulation cascades, metabolism, and oxidant detoxification. On the other hand, embryonic membrane proteins could respond to their endometrial secreted partners and orchestrate biological processes that are essential for conceptus survival and development, e.g., cell invasion, immune tolerance, and glycolysis. In 
addition, our in vitro and in vivo results of the physical interaction and physiological role of ALB-CLDN4cell adhesion also support the predictability of our constructed interaction map.

What's more interesting is that our constructed pathway network suggests a role of a metabolic shift in the conceptus that stimulates endometrial remodeling (Fig. 4H). During conceptus implantation, glycolysis upregulation leads to enhanced lactate production. The appropriate concentration of lactate at the maternal-fetal interface serves as an embryo-derived pregnancy recognition signal by promoting the histone lactylation modification in the endometrium, which might further participate in the regulation of redox homeostasis, apoptosis, cell proliferation, cell adhesion, and immune tolerance. Particularly, embryonic lactate improved the ROS concentration, glutathione metabolism, and apoptosis in the endometrium, and the GSH/GSSG also scavenged ROS to prevent excessive ROS damage to the endometrium.

Taken together, our findings identified many putative molecular and cellular mechanisms that might be essential for achieving successful embryo implantation. A more comprehensive understanding of conceptus-endometrium crosstalk at the protein level will provide important clues to develop clinical intervention strategies to improve pregnancy outcomes following both natural conception and assisted reproduction.

\section{Materials And Methods}

\section{Ethics statement}

The experiments were performed in accordance with the Guide for the Care and Use of Agricultural Animals in Agricultural Research and Teaching, and all procedures were approved by the Institutional Animal Care and Use Committee at the China Agricultural University (Beijing, China). Some publicly available data was analyzed to support our work. The endometrial proteomes of pregnant and pregnancy failed ewes during the peri-implantation period were obtained from Haichao Zhao et al. [42]. Gene expression in endometrial samples from women with and without endometriosis was obtained from GSE135485. The ChIP-seq data of histone lactylation were from GSE115354 [58]. The RNA-seq data of the endometrial luminal epithelium, endometrial glandular epithelium, and conceptus from ewes on day of pregnancy 12, 14, 16 and 20 were from GSE87017 [45]. The RNA-seq data of the pre-receptive to receptive human endometrium were from $\mathrm{S}$. Hu, et al. [71].

\section{Animals and treatment.}

Chinese Small Tail Han ewes with normal estrous cycles were selected for the present study. The procedures of estrous synchronization, superovulation, artificial insemination (Al), and transfer of goodquality blastocysts were performed as described in our previous study [42].

ICR female mice aged 7-8 week and ICR male mice aged 10-12 week were fed ad libitum 
and housed under controlled lighting conditions (12 light:12 dark). They were maintained under specific pathogen-free conditions. All animal experiments were approved by and performed in accordance with the guidelines of the Institutional Animal Care and Use Committee of China Agricultural University.

\section{Sample collection}

We collected good-quality embryos from thirty donors at day 6.5 of pregnancy. Then, two well-developed blastocysts were transferred into each synchronized recipient ewe (forty-eight synchronized ewes). Sampling procedures were similar to the methods detailed in our previous study [42]. Briefly, all recipients were slaughtered at day 17 of pregnancy, then their uteri were collected and the conceptuses were flushed out using phosphate-buffered saline (PBS). Thirty-seven recipients had filamentous conceptuses. The endometrial caruncular (C) areas and intercaruncular (IC) areas were collected and processed as described by Attia et al. [21]. Opening the ipsilateral uterine horn longitudinally by scissors, the $C$ areas were carefully cut out and collected, and then the IC areas were sampled. These samples were stored at liquid nitrogen until further analysis (Fig. 1A).

\section{Protein extraction}

We divided thirty-six samples into three equally pools, with twelve samples in each pool (Fig. 1A). Each pool was ground to powder in liquid nitrogen and stored overnight at $-20^{\circ} \mathrm{C}$ after adding a five-fold volume of chilled acetone containing 10\% trichloroacetic acid (TCA) and $10 \mathrm{mmol} / \mathrm{L}$ dithiothreitol (DTT). The samples were then centrifuged at $4^{\circ} \mathrm{C}, 16,000 \times \mathrm{g}$ for $20 \mathrm{~min}$ and the supernatant was discarded. The precipitates were mixed with $1 \mathrm{~mL}$ of chilled acetone containing $10 \mathrm{mmol} / \mathrm{L} \mathrm{DTT}$, stored for $30 \mathrm{~min}$ at $20^{\circ} \mathrm{C}$, and centrifuged at $4^{\circ} \mathrm{C}, 20,000 \times g$ for $30 \mathrm{~min}$. Centrifugation was repeated several times until the supernatant was colorless. The pellets were air-dried, dissolved in lysis buffer containing $1 \mathrm{mmol} / \mathrm{L}$ phenylmethanesulfonyl fluoride (PMSF), $2 \mathrm{mmol} / \mathrm{L}$ ethylenediaminetetraacetic acid (EDTA), and 10 $\mathrm{mmol} / \mathrm{L}$ DTT and sonicated at $200 \mathrm{~W}$ for $15 \mathrm{~min}$ before being centrifuged at 30,000 $\times \mathrm{g}$ at room temperature for $30 \mathrm{~min}$. The protein concentration in the supernatant was then detected by using the Bradford method.

\section{Peptide digestion}

Proteins $(50 \mu \mathrm{g})$ were taken from each sample, and isopycnic samples were prepared by adding $8 \mathrm{~mol} / \mathrm{L}$ urea solution. To reduce disulfide bonds, the samples were incubated with $10 \mathrm{mmol} / \mathrm{L}$ DTT at $56^{\circ} \mathrm{C}$ for 1 $\mathrm{h}$, and then cysteine bonding was blocked using $55 \mathrm{mmol} / \mathrm{L}$ iodoacetamide (IAM) in a dark room for 45 min. Thereafter, each sample was diluted 8-fold with $50 \mathrm{mmol} / \mathrm{L}$ ammonium bicarbonate and digested with Trypsin Gold at a protein: trypsin ratio of $20: 1$ at $37^{\circ} \mathrm{C}$ for $16 \mathrm{~h}$. Following desalting using a Strata $X$ C18 column (Phenomenex, Torrance, CA, USA), the samples were vacuum dried. Peptides generated from digestion were directly loaded for liquid chromatography-electrospray ionization tandem mass spectroscopy (LC-ESI-MS/MS) analysis.

\section{LC-ESI-MS/MS analysis with a linear ion trap-orbitrap (LTQ- orbitrap) collision induced dissociation (CID)}


Each sample was resuspended in buffer A [ $2 \%$ acetonitrile (ACN), $0.1 \%$ formic acid (FA)] and centrifuged at $20,000 \times g$ for $10 \mathrm{~min}$. The final peptide concentration for each sample was approximately $0.5 \mu \mathrm{g} / \mathrm{mL}$. The digested samples were fractionated using a Shimadzu LC-20AD nano-high performance liquid chromatography (HPLC) system (Shimadzu, Kyoto, Japan). Each sample $(10 \mu \mathrm{L})$ was loaded by the autosampler onto a $2 \mathrm{~cm}$ C18 trap column ( $200 \mu \mathrm{m}$ inner diameter), and the peptides were eluted onto a resolving $10 \mathrm{~cm}$ analytical $\mathrm{C} 18 \mathrm{column}$ ( $75 \mu \mathrm{m}$ inner diameter) prepared in-house. The samples were loaded at a flow rate of $15 \mu \mathrm{L} / \mathrm{min}$ for $4 \mathrm{~min}$, and then a 91 min gradient from $2-35 \%$ buffer $B(98 \% A C N$, $0.1 \% \mathrm{FA}$ ) was run at a flow rate of $400 \mathrm{~nL} / \mathrm{min}$, followed by a 5 min linear gradient to $80 \%$ buffer $B$ that was maintained for 8 min before finally returning to $2 \%$ buffer B within 2 min. The peptides were subjected to nano-electrospray ionization and then detected by MS/MS in an LTQ Orbitrap Velos (Thermo Fisher Scientific, Bremen, Germany) coupled online to an HPLC system. Intact peptides were detected in the Orbitrap analyzer at a resolution of $60,000 \mathrm{~m} / \mathrm{z}$. Peptides were selected for MS/MS using the CID operating mode with a normalized collision energy setting of $35 \%$, and ion fragments were detected in the LTQ. One MS scan followed by ten MS/MS scans was applied for the ten most abundant precursor ions above a threshold ion count of 5,000 in the MS survey scan. Dynamic exclusion was used, with the following parameters: Repeat counts $=2$; repeat duration $=30 \mathrm{~s}$; and exclusion duration $=120 \mathrm{~s}$. The applied electrospray voltage was $1.5 \mathrm{kV}$. Automatic gain control (AGC) was used to prevent overfilling of the ion trap; $1 \times 10^{4}$ ions were accumulated in the ion trap to generate CID spectra. For MS scans, the $\mathrm{m} / \mathrm{z}$ scan range was 350 to $2,000 \mathrm{Da}$.

\section{Proteomic analysis}

MaxQuant software (version 1.1.1.36) was used to analyze the mass spectra. Bos taurus is the most robust and extensive protein annotated species with a genomic database, with a strong homology to sheep $[75,87]$. Therefore, we generated one reference protein database by integrating the following databases and sequences of cattle proteins and limited publicly available sheep proteins, and removed duplicate proteins: GenBank nr (20110403), UniProt cow proteins (20110503), sheep proteins (http://www.livestockgenomics.csiro.au/sheep/), and cow proteins

(http://genomes.arc.georgetown.edu/drupal/bovine/). The MS/MS data were searched against the reference protein database using the search engine embedded in MaxQuant. Up to two missed cleavages were allowed. The first search was set to $20 \mathrm{ppm}$, and the MS/MS tolerance for CID was set to $0.5 \mathrm{Da}$. The false discovery rate (FDR) was set to 0.01 for peptide and protein identifications, which was estimated based on the fraction of reverse protein hits $[88,89]$. Proteins were considered identified when at least two peptides were identified, at least one of which was uniquely assignable to the corresponding sequence. In the case of identified peptides that were all shared between two proteins, these were combined and reported as one protein group. To control the false match frequency, the contents of the protein table were filtered to eliminate identifications from the reverse database and common contaminants $[90,91]$. The minimum peptide length was set to six amino acids. To perform label-free quantification analysis, the MaxQuant software suite containing an algorithm based on the extracted ion currents (XICs) of the peptides was used. Xcalibur 2.1 (Thermo Scientific) was used as quality control 
program to check the quality of chromatographs. This specific label-free processing method was performed as described by Waanders et al. [92].

\section{Cell culture}

A human endometrial cancer cell line (Ishikawa, ATCC, Manassas, VA, USA). Ishikawa cells were grown at $37^{\circ} \mathrm{C}$ in DMEM/F-12, HEPES (Gbico, UK) supplemented with $10 \%$ fetal bovine serum (FBS; Hyclone, Logan, UT, USA) and 1\% penicillin/streptomycin (Invitrogen, Waltham, MA, USA) in a humidified $5 \% \mathrm{CO}_{2}$ incubator.

\section{Western blotting}

Samples of acid extracted histones from IC areas, C areas, and Ishikawa cells were fractionated with 15\% sodium dodecyl sulfate-polyacrylamide gel electrophoresis and transferred to polyvinylidene fluoride membranes. The membranes were blocked with $5 \%$ skimmed milk in Tris buffered saline-Tween for one hour, followed by probing with anti-H3K18la (1:2000, PTM-1406, PTM Bio Inc., Hangzhou, China), anti-H3 (1:1000, ab12079, Abcam, Cambridge, MA, USA), anti-CLDN4 (1:500, sc-376643, Santa Cruz Biotechnology), or anti-ALB (1:500, sc-271605, Santa Cruz Biotechnology). After incubation at $4^{\circ} \mathrm{C}$ overnight, the membrane was probed with horseradish peroxidase-conjugated secondary antibody for one hour. After washing the blots were visualized using the ECL system (Bio-Rad, Hercules, CA, USA). The grey level of each bands was calculated by ImageJ (https://imagej.nih.gov/ij/, NIH, Bethesda, MD, USA).

\section{The detection of lactate concentration}

The lactate concentration in tissues was detected using a lactate assay Kit (MAK064, Sigma, St. Louis, MO, USA). The total protein concentration was quantified using an enhanced BCA protein assay kit (Beyotime Biotechnology, Jiangsu, China). Finally, the lactate concentration was normalized by the total protein concentration.

\section{The detection of GSH/GSSG}

Cells were harvested and washed three times with PBS. Then, the concentrations of GSH and GSSG were quantified using a GSH and GSSG Assay Kit following the manufacturer's instruction (Beyotime Institute of Biotechnology, Nanjing, China). Each experiment was performed with three replicates.

\section{Measurement of ROS}

The intracellular ROS levels of Ishikawa cells were determined using a Reactive Oxygen Species Assay Kit (Beyotime Institute of Biotechnology), according to the manufacturer's instruction. The fluorescence signals were imaged using a confocal laser scanning microscope (Digital Eclipse C1; Nikon). The fluorescent intensity was calculated using ImageJ.

\section{Cell viability assay}

Ishikawa cells were resuspended and seeded into 96-well culture plates at a density of 2,000 cells per well. Then cells were treated with $10 \mathrm{mM}$ sodium L-lactate for 12 hours and 24 hours. Cell proliferation 
was detected using an Enhanced Cell Counting Kit-8 (CCK-8) (Beyotime).

\section{The detection of cell apoptosis}

A terminal deoxynucleotidyl transferase mediated dUTP nick end-labeling (TUNEL) assay was used to analyze cell apoptosis, following the manufacturer's instruction of the In Situ Cell Death Detection Kit (11684817910, Roche Molecular Biochemicals, Indianapolis, IN, USA). Ishikawa cells were seeded into 24well culture plates, and treated with $10 \mathrm{mM}, 50 \mathrm{mM}$, or $100 \mathrm{mM}$ sodium L-lactate for 24 hours. The fluorescence signals were imaged using a confocal laser scanning microscope (Digital Eclipse C1; Nikon). The fluorescent intensity was calculated using ImageJ.

\section{Cell adhesion assay}

Fibronectin $\left(10 \mu \mathrm{g} / \mathrm{mL}\right.$, Sigma) was used to coat a $35-\mathrm{mm}$ culture dish, overnight at $4^{\circ} \mathrm{C}$, and then washed three times with PBS. Ishikawa cells were resuspended in serum-free medium (DMEM/F-12, HEPES (Gbico, UK) supplemented with $1 \%$ penicillin/streptomycin (Invitrogen)) and $5 \mu \mathrm{g} / \mathrm{mL}, 25 \mu \mathrm{g} / \mathrm{mL}, 100$ $\mu \mathrm{g} / \mathrm{mL}$ BSA (Bovine serum albumin, Sigma) and then plated in the 35-mm dishes. After 2 hours of incubation, the non-adherent cell containing media was aspirated off and each well was washed gently with PBS. Then, images of adherent cells were acquired under a microscope (Olympus) from at least ten random fields.

\section{Preparation of mouse embryos}

All experiments involved in embryo preparation were performed as previously described [93], with minor modifications. ICR female mice were superovulated by intraperitoneal injection of $5 \mathrm{IU}$ pregnant mare serum gonadotropin and a further intraperitoneal injection $48 \mathrm{~h}$ later of $5 \mathrm{IU}$ human chorionic gonadotropin (hCG). Then sperm were obtained from the cauda epididymis and capacitated for $1 \mathrm{~h}$ in human tubal fluid (HTF; SAGE) medium at $37^{\circ} \mathrm{C}$ in $5 \% \mathrm{CO} 2$. Oocytes were collected from the ampullae at $14 \mathrm{~h}$ after hCG treatment. Gametes were then co-incubated in HTF medium for $4 \mathrm{~h}$ at $37^{\circ} \mathrm{C}$ in $5 \% \mathrm{CO} 2$. After $4 \mathrm{~h}$ in the incubator, zygotes were washed and cultured to the blastocyst stage in potassium simplex optimization medium containing amino acids (KSOM + AA; Millipore) under mineral oil at $37^{\circ} \mathrm{C}$ in $5 \% \mathrm{CO} 2$.

\section{In vitro embryo implantation model and mouse embryo attachment assay}

The in vitro embryo implantation model was constructed according to the previous reports $[59,60]$. For attachment assay, well-developed mouse blastocysts were collected and then transferred onto differently treated Ishikawa cells in serum-free medium, with ten blastocysts per well. After the blastocysts and Ishikawa cells were cocultured for 24 or 48 hours, a standardized plate movement protocol was implemented to measure the number of attached embryos [94, 95].

\section{Fluorescent immunocytochemistry}

Ishikawa cells were co-cultured with blastocysts 72 hours, then fixed with $4 \%$ paraformaldehyde for further detection of H3K18la. For albumin overload assay in Fig. 2C and Fig. S5B, control group was incubated 30 min with FBS-medium (DMEM/F-12, HEPES supplemented with 1\% penicillin/streptomycin 
and $10 \%$ FBS), while MSA group were incubated with MS-medium (DMEM/F-12, HEPES (Gbico, UK) supplemented with 1\% penicillin/streptomycin (Invitrogen)) and 10\% mouse serum) 30 min, then fixed with $4 \%$ paraformaldehyde for further detection of ALB and CLDN4. Immunostaining was performed according to standard protocols using the following primary antibodies: anti-H3K18la (1:1000, PTM-1406, PTM Bio Inc.), anti-ALB (1:250, sc-271605, Santa Cruz Biotechnology), anti-CLDN4 (1:250, 16195-1-AP, Proteintech). And appropriate Alexa Fluor dye conjugated secondary antibodies (Invitrogen) were used. Nuclei were stained with DAPI (Life Technologies). The fluorescence signals were imaged using a confocal laser scanning microscope (Digital Eclipse C1; Nikon). Data analysis was performed by ImageJ.

\section{Intrauterine injection}

ICR female mice were superovulated by intraperitoneal injection of $5 \mathrm{IU}$ pregnant mare serum gonadotropin and a further intraperitoneal injection $48 \mathrm{~h}$ later of $5 \mathrm{IU}$ human chorionic gonadotropin (hCG). Each female mouse was caged with one male and allowed to mate naturally overnight. Day 1 of pregnancy was designated as the next morning when a vaginal plug was formed. On day 4 of pregnancy, each uterine horn of female mice was slowly injected with $5 \mu \mathrm{L}$ of saline (or $10 \mathrm{mg} / \mathrm{mL}, 25 \mathrm{mg} / \mathrm{mL}$ oxamate, or $10 \mathrm{mg} / \mathrm{mL}$ oxamate with $0.04 \mathrm{mM}, 0.2 \mathrm{mM}, 1 \mathrm{mM}, 5 \mathrm{mM}$ lactate) using a 26-gauge Hamilton syringe (no. A6410, Sigma). In the morning of day 5 , the treated animals were sacrificed to count the numbers of implantation sites [96].

\section{Analyses of differentially abundant proteins}

To facilitate data analysis, all proteins were mapped to the Ensembl Bos Taurus gene ID. P values from student's t-test were corrected for multiple hypothesis tests using the false discovery rate (FDR) procedure [97]. For each comparison, gene expression levels were considered significantly different when FDR $<0.05$ and fold change $(F C)>2$. The protein quantification values of the conceptus, $C$ area, IC area, and DAPs of each comparison (conceptus vs. C area and conceptus vs. IC area) are shown in Table. S1.

\section{Annotations of differentially abundant membrane and secreted proteins}

The annotations of membrane and secreted proteins were processed as described by Vento-Tormo et al [35]. Briefly, DAPs were mapped to UniProt (https://www.uniprot.org/), then KW-0964 (secreted) was used to screen out the secreted partners. KW-1003 (cell membrane) was used to screen out the plasma membrane proteins. Peripheral proteins from the plasma membrane were annotated using the UniProt Keyword SL-9903, and the remaining proteins were annotated as membrane proteins, which act as extracellular signal receptors. Interestingly, some proteins were annotated as both secreted proteins and membrane proteins, such as heat shock protein 90 alpha family class B member 1 (HSP90AB1) and elastin microfibril interfacer 1 (EMILIN1). The differentially abundant secreted proteins or membrane proteins are shown in Table S2. Phenotype annotations of differentially abundant membrane proteins or secreted proteins were analyzed based on the MGI database (Mouse Genome Informatics, http://www.informatics.jax.org/phenotypes.shtml). 


\section{Construction of membrane-secreted partner interactions}

We used the Search Tool for the Retrieval of Interacting Genes/Proteins (STRING version 11.0; https://string-db.org/ [98]) to build the membrane-secreted partner interactions using edge information from three separate forms of evidence: Databases, experiments, and text mining. Firstly, we inputted a secreted protein (or a membrane protein) to acquire its interacting partners and interaction scores. Then we mapped its interacting partners to UniProt to screen out the membrane partners (or secreted partners). Finally, we chose the membrane partner (or secreted partner) with the highest interaction score. In this way, we constructed the interactions of differentially abundant membrane proteins (or secreted proteins) with their secreted partners (or membrane partners). All the interactions are shown in Table S3.

\section{Gene ontology (GO) and KEGG pathway analysis and pathway network construction}

DAVID version 6.8 (https://david.ncifcrf.gov/home.jsp) enables the generation of specific functional annotations of biological processes affected by treatment from the target gene lists produced in highthroughput experiments $[99,100]$. We used DAVID to carry the gene-annotation enrichment analysis to obtain a functional view of the DAPs. The genetic background in DAVID is Bos Taurus, which was a default parameter given by DAVID when we uploaded the DAPs list. Visualizations of significant $G O$ and Kyoto Encyclopedia of Genes and Genomes (KEGG) categories $(P$ values $<0.05)$ was carried by the "ggplot2" package [101] in R (R version 3.5.1. https://www.R-project.org/.). The pathway network construction and key pathway findings were determined using PathwayConnector (PC) (http://bioinformatics.cing.ac.cy/PathwayConnector/\#) [47].

Hierarchical clustering analysis, Principal Components Analysis (PCA), Gene Set Enrichment Analysis (GSEA), and protein-protein network construction

Unsupervised hierarchical clustering analysis was carried using the "hclust" function in R. PCA of all samples was carried using the "Prcomp" function in R. Significantly differentially regulated pathways were identified by GSEA (NES > 1, FDR < 0.25) $[102,103]$. We used STRING to construct the protein-protein interaction network of DAPs, and then visualized using the cytoHubba plugin in the Cytoscape software according to the degree number [104].

\section{Constructed the protein-protein docking model of ALB- CLDN4}

The crystal structure of ALB (1A06) and CLDN4 (7KP4) was downloaded from protein data bank (http://www.rcsb.org/). And the ClusPro (https://cluspro.org) was used for protein-protein docking [105108]. We chose the balanced pattern to predict the interaction of ALB and CLDN4, and cluster scores were used to evaluated the docking model. Then the PyMOL software was used to show the docking model with highest score as cartoon and surface shapes.

\section{Co-immunoprecipitation assay}


Samples were fractionated using RIPA (beyotime) supplemented with protease and phosphatase inhibitors (beyotime), and used for immunoprecipitation using Dynabeads protein $\mathrm{G}$ according to manufacturers' protocol (10007D, Life technologies). CLDN4 (1 $\mu \mathrm{g} / 1 \mathrm{mg}$ lysate, 16195-1-AP, Proteintech) and IgG antibody (rabbit anti-mouse, 06-371, Sigma) were used for immunoprecipitation. At the final step, the beads-antibody-antigen complexes were eluted in $50 \mu \mathrm{L}$ loading buffer $(2 \times$ Laemmli Sample Buffer (Bio-Rad), 5\% 2-Mercaptoethanol (Sigma)), and boiled at $70^{\circ} \mathrm{C}$ for $10 \mathrm{~min}$. The beads were separated from the magnet and supernatant (containing the eluted protein) and used for western blotting.

\section{Real-time quantitative PCR analysis}

Total RNAs from the mouse E6.5 embryo and decidua tissues were extracted using TRIzol reagent (Invitrogen). cRNA was reverse-transcribed into complementary DNA (cDNA) with a HiScript II reverse transcriptase reagent kit (Vazyme, Nanjing, China). Real-time PCR was performed using SsoFast EvaGreen Supermix (BioRad) in a CFX96 real-time PCR machine (Bio-Rad). Actb was used as internal reference. At least three independent experiments were performed. Primers used for qPCR: $A c t b-\mathrm{F}$ : TGGCGCTTTTGACTCAGGAT, Actb-R: GGGATGTTTGCTCCAACCAA; $A l b$-F:

GAAAACCAGGCGACTATCTCCA; Alb-R: TGCACACTTCCTGGTCCTCA; Cldn4-F: CGCTACTCTTGCCATTACGC, Cldn4-R: TCACTCAGCACACCATGACTTG.

\section{Fluorescent immunohistochemistry}

Fixed E6.5 tissues were prepared according to standard protocols for paraffin embedding. The paraffinembedded tissues were sectioned serially at $5 \mu \mathrm{m}$ thick. The sections were microwaved in the antigen unmasking solution, blocked with bovine serum albumin solution, and incubated overnight with anti-ALB (1:200, sc-271605, Santa Cruz Biotechnology) and anti-CLDN4 (1:200, 16195-1-AP, Proteintech) at $4^{\circ} \mathrm{C}$. In the following day, slides were washed in PBS containing $0.1 \%$ Tween 20 (PBS-T), incubated with secondary antibodies conjugated with Alexa Fluor 488 (anti-mouse; Invitrogen) or Alexa Fluor 594 (antirabbit; Invitrogen) for $1 \mathrm{~h}$ at room temperature, washed with PBS-T, counterstained with DAPI and mounted. The fluorescence signals were imaged using a confocal laser scanning microscope (Digital Eclipse C1; Nikon). Data analysis was performed by ImageJ.

\section{Statistical analysis}

The $P$ value of Student's t-test was calculated using GraphPad Prism 7.0 software (GraphPad Inc., La Jolla, CA, USA) or R for individual analysis. It was considered significant when the $P$ value $<0.05$. Error bars represent the means \pm SEM (Standard Error of the Mean). Details of individual tests are outlined within each figure legend.

\section{Declarations}

\section{Data Availability:}

All relevant data are within the paper and its Supporting Information files. 


\section{Competing interests}

The authors declare that they have no competing interests.

\section{Funding}

This work was supported by the grants from National Key R\&D Program (2017YFD0501905 and 2017YFD0501901), National Natural Science Foundation of China (No.3167246 and 31972573 ), National Support Program for Youth Top-notch Talents, and the Earmarked Fund for the Innovative Teams of Beijing Swine Industrialization Research Program, National transgenic major program (2009ZX08006008B and 2008ZX08006-002, 2011ZX08006-002, 2013ZX08006-002).

\section{Acknowledgements}

We thank the members of our laboratory for their helpful comments on the manuscript.

\section{Author contributions}

Conceptualization: Qianying Yang, Lei An, Jianhui Tian

Resources: Kai Miao, Haichao Zhao,

Formal Analysis: Qianying Yang, Juan Liu, and Lei An, Wei Zhao

Funding Acquisition: Jianhui Tian and Lei An

Investigation: Qianying Yang, Juan Liu, Yue Wang, Wei Zhao, Wenjing Wang, Jian Cui, Jiajun Yang, Yuan Yue, Meiqiang Chu, Qingji Lyu, Lizhu Ma, Yawen Tang, Yupei Hu

Writing - Original Draft Preparation: Qianying Yang

Writing - Review \& Editing: Qianying Yang, Juan Liu, Lei An and Jianhui Tian

All authors read and approved the final manuscript.

\section{Qianying Yang and Juan Liu contributed equally to this work.}

\section{Abbreviations}

ACN: acetonitrile; AGC: automatic gain control; Al: artificial insemination; ART: assistant reproductive technology; ALPL: alkaline phosphatase, liver/bone/kidney; AHSG: alpha 2-HS glycoprotein; A1BG: alpha1-B glycoprotein; BCAM: basal cell adhesion molecule; BSG: basigin; BSA: bovine serum albumin; $C$ areas: caruncular areas; CID: collision induced dissociation; C5: complement C5; C9: complement C9; CAPN2: calpain 2; CSTB: cathepsin B; CHP1: calcineurin like EF-hand protein 1; CLDN4: claudin 4; COL6A1: collagen type $\mathrm{VI}$ alpha 1 chain; COL6A2: collagen type VI alpha 2 chain; DTT: dithiothreitol; DEPs: 
differentially expressed proteins; EMILIN1: elastin microfibril interfacer 1; EMB: embigin; EDTA: ethylenediaminetetraacetic acid; ESI: electrospray ionization; FBS: fetal bovine serum; FDR: false discovery rate; FC: fold change; FITC: fluorescein isothiocyanate labeled; FA: formic acid; FN1: fibronectin 1; FKBP1A: FK506 binding protein 1A; GE: glandular epithelium; GOT2: glutamic-oxaloacetic transaminase 2; GO: Gene Ontology; GSEA: Gene Set Enrichment Analysis; HSP90AB1: heat shock protein 90 alpha family class B member 1; HSPA8: heat shock protein family A (Hsp70) member 8; HPLC: High Performance Liquid Chromatography; HE: hematoxylin-eosin; IVO: in vivo; IVF: in vitro fertilization; IC areas: intercaruncular areas; IAM: iodoacetamide; IFN-t: interferon $\tau$; ISG15: Interferon-Stimulated Gene15; IDH2: Isocitrate Dehydrogenase (NADP(+)) 2; ITGA9: integrin subunit alpha 9; LC: liquid chromatography; LTQ: linear ion trap; LE: luminal epithelium; LC-ESI-MS/MS: liquid chromatographyelectrospray ionization-tandem mass spectroscopy; MCT1: monocarboxylate transporter 1; MCTs: monocarboxylate transporters; MS: mass spectrometry; myo: myometrium; NME1: nometastatic gene 23H1; P/S: Penicillin and streptomycin; PLG: plasminogen; PTK7: protein tyrosine kinase 7; PC: PathwayConnector; PBS: phosphate-buffered saline; PMSF: phenylmethanesulfonyl fluoride; PCA: principal component analysis; PGF2a: prostaglandin F2a; RPSA: 40S ribosomal protein SA; ROS: reactive oxygen species; SCNT: somatic cell nuclear transfer; SLC2A1: solute carrier family 2 member 1; S: stroma; TCA: trichloroacetic acid; TGFBI: transforming growth factor beta induced; XICs: extracted ion currents; v: blood vessels;

\section{References}

1. Wang H, Dey SK. Roadmap to embryo implantation: clues from mouse models. Nat Rev Genet 2006; 7:185-199.

2. Bazer FW. Uterine receptivity to implantation of blastocysts in mammals. Front Biosci 2011; S3:184.

3. Cha J, Sun X, Dey SK. Mechanisms of implantation: strategies for successful pregnancy. Nat Med 2012; 18:1754-1767.

4. Zinaman MJ, Clegg ED, Brown CC, O'Connor J, Selevan SG. Estimates of human fertility and pregnancy loss. Fertil Steril 1996; 65:503-509.

5. Norwitz ER, Schust DJ, Fisher SJ. Implantation and the Survival of Early Pregnancy. N Engl J Med $2001 ; 345: 1400-1408$.

6. Edwards RG. Human implantation: the last barrier in assisted reproduction technologies? Reprod Biomed Online 2006; 13:887-904.

7. Zhang S, Lin H, Kong S, et al. Physiological and molecular determinants of embryo implantation. Mol Aspects Med 2013; 34:939-980.

8. Morel O, Laporte-Broux B, Tarrade A, Chavatte-Palmer P. The use of ruminant models in biomedical perinatal research. Theriogenology 2012; 78:1763-1773.

9. Lee KY, DeMayo FJ. Animal models of implantation. Reproduction 2004; 128:679-695.

10. Martal JL, Chêne NM, Huynh LP, et al. IFN-tau: A novel subtype I IFN1. Structural characteristics, nonubiquitous expression, structure-function relationships, a pregnancy hormonal embryonic signal and 
cross-species therapeutic potentialities. Biochimie 1998; 80:755-777.

11. Bazer FW. Mediators of Maternal Recognition of Pregnancy in Mammals. Exp Biol Med 1992; 199:373-384.

12. Spencer TE, Johnson GA, Bazer FW, Burghardt RC. Implantation mechanisms: Insights from the sheep. Reproduction 2004; 128:657-668.

13. Spencer TE, Johnson GA, Bazer FW, Burghardt RC, Palmarini M. Pregnancy recognition and conceptus implantation in domestic ruminants: roles of progesterone, interferons and endogenous retroviruses. Reprod Fertil Dev 2007; 19:65.

14. Choi Y, Johnson GA, Burghardt RC, et al. Interferon Regulatory Factor-Two Restricts Expression of Interferon-Stimulated Genes to the Endometrial Stroma and Glandular Epithelium of the Ovine Uterus1. Biol Reprod 2001; 65:1038-1049.

15. Rosenfeld CS, Han C-S, Alexenko AP, Spencer TE, Roberts RM. Expression of Interferon Receptor Subunits, IFNAR1 and IFNAR2, in the Ovine Uterus1. Biol Reprod 2002; 67:847-853.

16. Chen Y, Antoniou E, Liu Z, Hearne LB, Roberts RM. A microarray analysis for genes regulated by interferon- $\tau$ in ovine luminal epithelial cells. Reproduction 2007; 134:123-135.

17. Oliveira JF, Henkes LE, Ashley RL, et al. Expression of Interferon (IFN)-Stimulated Genes in Extrauterine Tissues during Early Pregnancy in Sheep Is the Consequence of Endocrine IFN-t Release from the Uterine Vein. Endocrinology 2008; 149:1252-1259.

18. Spencer TE, Bartol FF, Bazer FW, Johnson GA, Joyce MM. Identification and Characterization of Glycosylation-Dependent Cell Adhesion Molecule 1-Like Protein Expression in the Ovine Uterus. Biol Reprod 1999; 60:241-250.

19. Dorniak P, Welsh TH, Bazer FW, Spencer TE. Endometrial HSD11B1 and Cortisol Regeneration in the Ovine Uterus: Effects of Pregnancy, Interferon Tau, and Prostaglandins1. Biol Reprod 2012; 86.

20. Bauersachs S, Ulbrich SE, Zakhartchenko V, et al. The endometrium responds differently to cloned versus fertilized embryos. Proc Natl Acad Sci U S A 2009; 106:5681-6.

21. Mansouri-Attia N, Sandra O, Aubert J, et al. Endometrium as an early sensor of in vitro embryo manipulation technologies. Proc Natl Acad Sci 2009; 106:5687-5692.

22. Biase FH, Hue I, Dickinson SE, et al. Fine-tuned adaptation of embryo-endometrium pairs at implantation revealed by transcriptome analyses in Bos taurus. PLOS Bio/ 2019; 17:1-20.

23. Nie J, An L, Miao K, et al. Comparative analysis of dynamic proteomic profiles between in vivo and in vitro produced mouse embryos during postimplantation period. J Proteome Res 2013; 12:38433856.

24. Chen Z, Hagen DE, Elsik CG, et al. Characterization of global loss of imprinting in fetal overgrowth syndrome induced by assisted reproduction. Proc Natl Acad Sci U S A 2015; 112:4618-4623.

25. Tan K, An L, Miao K, et al. Impaired imprinted X chromosome inactivation is responsible for the skewed sex ratio following in vitro fertilization. Proc Natl Acad Sci 2016; 113:3197-3202. 
26. Malo Estepa I, Tinning H, Rosas Vasconcelos EJ, et al. Protein Synthesis by Day 16 Bovine Conceptuses during the Time of Maternal Recognition of Pregnancy. Int J Mol Sci 2020; 21:2870.

27. Wang Y, Wang C, Hou Z, et al. Comparative analysis of proteomic profiles between endometrial caruncular and intercaruncular areas in ewes during the peri-implantation period. J Anim Sci Biotechnol 2013; 4:39.

28. Moraes JGN, Behura SK, Geary TW, Spencer TE. Analysis of the uterine lumen in fertility-classified heifers: I. Glucose, prostaglandins, and lipids. Biol Reprod 2020; 102:456-474.

29. Moraes JGN, Behura SK, Bishop J V., Hansen TR, Geary TW, Spencer TE. Analysis of the uterine lumen in fertility-classified heifers: II. Proteins and metabolitest. Biol Reprod 2020; 102:571-587.

30. Biase FH, Rabel C, Guillomot M, et al. Massive dysregulation of genes involved in cell signaling and placental development in cloned cattle conceptus and maternal endometrium. Proc Natl Acad Sci US A 2016; 113:14492-14501.

31. Yang Q, Fu W, Wang Y, et al. The proteome of IVF-induced aberrant embryo-maternal crosstalk by implantation stage in ewes. J Anim Sci Biotechnol 2020; 11:7.

32. Moraes JGN, Behura SK, Geary TW, Hansen PJ, Neibergs HL, Spencer TE. Uterine influences on conceptus development in fertility-classified animals. Proc Natl Acad Sci 2018; 115:E1749-E1758.

33. Ramilowski JA, Goldberg T, Harshbarger J, et al. A draft network of ligand-receptor-mediated multicellular signalling in human. Nat Commun 2015; 6:7866.

34. Kumar MP, Du J, Lagoudas G, et al. Analysis of Single-Cell RNA-Seq Identifies Cell-Cell Communication Associated with Tumor Characteristics. Cell Rep 2018; 25:1458-1468.e4.

35. Vento-Tormo R, Efremova M, Botting RA, et al. Single-cell reconstruction of the early maternal-fetal interface in humans. Nature 2018; 563:347-353.

36. Spencer T., Burghardt R., Johnson G., Bazer F. Conceptus signals for establishment and maintenance of pregnancy. Anim Reprod Sci 2004; 82-83:537-550.

37. Wan P-C, Bao Z-J, Wu Y, et al. avß3 Integrin may Participate in Conceptus Attachment by Regulating Morphologic Changes in the Endometrium during Peri-implantation in Ovine. Reprod Domest Anim $2011 ; 46: 840-847$.

38. Song G, Satterfield MC, Kim J, Bazer FW, Spencer TE. Gastrin-Releasing Peptide (GRP) in the Ovine Uterus: Regulation by Interferon Tau and Progesterone1. Biol Reprod 2008; 79:376-386.

39. Bazer FW, Spencer TE, Ott TL. Interferon tau: A novel pregnancy recognition signal. Am J Reprod Immunol 1997; 37:412-420.

40. Igwebuike UM. A review of uterine structural modifications that influence conceptus implantation and development in sheep and goats. Anim Reprod Sci 2009; 112:1-7.

41. TABIBZADEH S. Human Endometrium: An Active Site of Cytokine Production and Action. Endocr Rev $1991 ; 12: 272-290$.

42. Zhao H, Sui L, Miao K, et al. Comparative analysis between endometrial proteomes of pregnant and non-pregnant ewes during the peri-implantation period. J Anim Sci Biotechnol 2015; 6:1-14. 
43. Singh $\mathrm{H}$, Aplin JD. Adhesion molecules in endometrial epithelium: Tissue integrity and embryo implantation. J Anat 2009; 215:3-13.

44. Leese HJ. The formation and function of oviduct fluid. Reproduction 1988; 82:843-856.

45. Brooks K, Burns GW, Moraes JGN, Spencer TE. Analysis of the Uterine Epithelial and Conceptus Transcriptome and Luminal Fluid Proteome During the Peri-Implantation Period of Pregnancy in Sheep. Biol Reprod 2016; 95:88-88.

46. Dobrinsky JR, Johnson LA, Rath D. Development of a Culture Medium (BECM-3) for Porcine Embryos: Effects of Bovine Serum Albumin and Fetal Bovine Serum on Embryo Development. Biol Reprod 1996; 55:1069-1074.

47. Minadakis G, Zachariou M, Oulas A, Spyrou GM. PathwayConnector: finding complementary pathways to enhance functional analysis. Wren J, ed. Bioinformatics 2019; 35:889-891.

48. Kelly A, West JD. Genetic evidence that glycolysis is necessary for gastrulation in the mouse. Dev Dyn 1996; 207:300-308.

49. Merkle S, Pretsch W. A glucosephosphate isomerase (GPI) null mutation in Mus musculus: evidence that anaerobic glycolysis is the predominant energy delivering pathway in early post-implantation embryos. Comp Biochem Physiol Part B Comp Biochem 1992; 101:309-314.

50. Kramer AC, Steinhauser CB, Gao H, et al. Steroids Regulate SLC2A1 and SLC2A3 to Deliver Glucose Into Trophectoderm for Metabolism via Glycolysis. Endocrinology 2020; 161:1-19.

51. Smith DG, Sturmey RG. Parallels between embryo and cancer cell metabolism. Biochem Soc Trans 2013; 41:664-669.

52. Gardner DK. Lactate production by the mammalian blastocyst: Manipulating the microenvironment for uterine implantation and invasion? BioEssays 2015; 37:364-371.

53. Hu W, Liang YX, Luo JM, et al. Nucleolar stress regulation of endometrial receptivity in mouse models and human cell lines. Cell Death Dis 2019; 10.

54. Gu XW, Yang Y, Li T, et al. ATP mediates the interaction between human blastocyst and endometrium. Cell Prolif 2019:1-11.

55. Gardner DK. Lactate production by the mammalian blastocyst: Manipulating the microenvironment for uterine implantation and invasion? BioEssays 2015; 37:364-371.

56. Belt JA, Thomas JA, Buchsbaum RN, Racker E. Inhibition of lactate transport and glycolysis in Ehrlich ascites tumor cells by bioflavonoids. Biochemistry 1979; 18:3506-3511.

57. Payen VL, Mina E, Van Hée VF, Porporato PE, Sonveaux P. Monocarboxylate transporters in cancer. Mol Metab 2020; 33:48-66.

58. Zhang D, Tang Z, Huang $\mathrm{H}$, et al. Metabolic regulation of gene expression by histone lactylation. Nature 2019; 574:575-580.

59. Ruane PT, Berneau SC, Koeck R, et al. Apposition to endometrial epithelial cells activates mouse blastocysts for implantation. Mol Hum Reprod 2017; 23:617-627. 
60. Berneau SC, Ruane PT, Brison DR, Kimber SJ, Westwood M, Aplin JD. Investigating the role of CD44 and hyaluronate in embryo-epithelial interaction using an in vitro model. Mol Hum Reprod 2019; 25:265-273.

61. Leitao B, Jones MC, Fusi L, et al. Silencing of the JNK pathway maintains progesterone receptor activity in decidualizing human endometrial stromal cells exposed to oxidative stress signals. FASEB J 2010; 24:1541-1551.

62. Wu J, Hansen JM, Hao L, Taylor RN, Sidell N. Retinoic acid stimulation of VEGF secretion from human endometrial stromal cells is mediated by production of reactive oxygen species. J Physiol $2011 ;$ 589:863-875.

63. Hayes JD, Dinkova-Kostova AT. The Nrf2 regulatory network provides an interface between redox and intermediary metabolism. Trends Biochem Sci 2014; 39:199-218.

64. Armant DR. Life and death responses to trophinin-mediated adhesion during blastocyst implantation. Cell Cycle 2011; 10:579-578.

65. Al-Gubory $\mathrm{KH}$, Garrel C. Antioxidative signalling pathways regulate the level of reactive oxygen species at the endometrial-extraembryonic membranes interface during early pregnancy. Int $J$ Biochem Cell Biol 2012; 44:1511-1518.

66. Al-Gubory KH, Faure P, Garrel C. Different enzymatic antioxidative pathways operate within the sheep caruncular and intercaruncular endometrium throughout the estrous cycle and early pregnancy. Theriogenology 2017; 99:111-118.

67. Canestrari F, Buoncristiani U, Galli F, et al. Redox state, antioxidative activity and lipid peroxidation in erythrocytes and plasma of chronic ambulatory peritoneal dialysis patients. Clin Chim Acta 1995; 234:127-136.

68. Kim Y-J. Antimelanogenic and Antioxidant Properties of Gallic Acid. Biol Pharm Bull 2007; 30:10521055.

69. Sui L, An L, Tan K, et al. Dynamic Proteomic Profiles of In Vivo- and In Vitro-Produced Mouse Postimplantation Extraembryonic Tissues and Placentas1. Biol Reprod 2014; 91:1-16.

70. Carson DD, Lagow E, Thathiah A, et al. Changes in gene expression during the early to mid-luteal (receptive phase) transition in human endometrium detected by high-density microarray screening. Mol Hum Reprod 2002; 8:871-9.

71. Hu S, Yao G, Wang Y, et al. Transcriptomic changes during the pre-receptive to receptive transition in human endometrium detected by RNA-SEq. J Clin Endocrinol Metab 2014; 99:E2744-E2753.

72. Hartshorn C, Rice JE, Wangh LJ. Differential pattern of Xist RNA accumulation in single blastomeres isolated from 8-cell stage mouse embryos following laser zona drilling. Mol Reprod Dev 2003; 64:41-51.

73. McCarrey JR, Watson C, Atencio J, et al. X-chromosome inactivation during spermatogenesis is regulated by an Xist/Tsix-independent mechanism in the mouse. Genesis 2002; 34:257-266.

74. Bergh T, Ericson A, Hillensjö T, Nygren KG, Wennerholm UB. Deliveries and children born after in-vitro fertilisation in Sweden 1982-95: A retrospective cohort study. Lancet 1999. 
75. Romero JJ, Liebig BE, Broeckling CD, Prenni JE, Hansen TR. Pregnancy-induced changes in metabolome and proteome in ovine uterine flushings. Biol Reprod 2017; 97:273-287.

76. Kaneko Y, Murphy CR, Day ML. Calpain 2 activity increases at the time of implantation in rat uterine luminal epithelial cells and administration of calpain inhibitor significantly reduces implantation sites. Histochem Cell Biol 2014; 141:423-430.

77. Lessey BA. Adhesion molecules and implantation. J Reprod Immunol 2002; 55:101-112.

78. Xie KM, Hou XF, Li MQ, Li DJ. NME1 at the human maternal-fetal interface downregulates titin expression and invasiveness of trophoblast cells via MAPK pathway in early pregnancy. Reproduction 2010; 139:799-808.

79. Li MQ, Shao J, Meng YH, et al. NME1 suppression promotes growth, adhesion and implantation of endometrial stromal cells via Akt and MAPK/Erk1/2 signal pathways in the endometriotic milieu. Hum Reprod 2013; 28:2822-2831.

80. Quinn CE, Simmons DG, Kennedy TG. Expression of Cystatin C in the rat endometrium during the peri-implantation period. Biochem Biophys Res Commun 2006; 349:236-244.

81. Song G, Bailey DW, Dunlap KA, et al. Cathepsin B, Cathepsin L, and Cystatin C in the Porcine Uterus and Placenta: Potential Roles in Endometrial/Placental Remodeling and in Fluid-Phase Transport of Proteins Secreted by Uterine Epithelia Across Placental Areolae1. Biol Reprod 2010; 82:854-864.

82. Simon C. Embryonic Regulation of Integrins 3, 4, and 1 in Human Endometrial Epithelial Cells in Vitro. J Clin Endocrinol Metab 1997; 82:2607-2616.

83. Wadehra M, Forbes A, Pushkarna N, et al. Epithelial membrane protein-2 regulates surface expression of avß3 integrin in the endometrium. Dev Biol 2005; 287:336-345.

84. Goossens K, Van Soom A, Van Zeveren A, Favoreel H, Peelman LJ. Quantification of Fibronectin 1 (FN1) splice variants, including two novel ones, and analysis of integrins as candidate FN1 receptors in bovine preimplantation embryos. BMC Dev Biol 2009; 9:1-16.

85. George EL, Georges-Labouesse EN, Patel-King RS, Rayburn H, Hynes RO. Defects in mesoderm, neural tube and vascular development in mouse embryos lacking fibronectin. Development 1993; 119:1079-1091.

86. Seguinot-Tarafa I, Luna N, Suarez E, Appleyard CB, Flores I. Inhibition of Histone Methyltransferase EZH2 Suppresses Endometriotic Vesicle Development in a Rat Model of Endometriosis. Reprod Sci 2020; 27:1812-1820.

87. Koch JM, Ramadoss J, Magness RR. Proteomic profile of uterine luminal fluid from early pregnant ewes. J Proteome Res 2010; 9:3878-85.

88. Cox J, Mann M. MaxQuant enables high peptide identification rates, individualized p.p.b.-range mass accuracies and proteome-wide protein quantification. Nat Biotechnol 2008; 26:1367-1372.

89. Graumann J, Hubner NC, Kim JB, et al. Stable Isotope Labeling by Amino Acids in Cell Culture (SILAC) and Proteome Quantitation of Mouse Embryonic Stem Cells to a Depth of 5,111 Proteins. Mol Cell Proteomics 2008; 7:672-683. 
90. Feng J, Naiman DQ, Cooper B. Probability-based pattern recognition and statistical framework for randomization: Modeling tandem mass spectrum/peptide sequence false match frequencies. Bioinformatics 2007; 23:2210-2217.

91. Li G-Z, Vissers JPC, Silva JC, Golick D, Gorenstein M V., Geromanos SJ. Database searching and accounting of multiplexed precursor and product ion spectra from the data independent analysis of simple and complex peptide mixtures. Proteomics 2009; 9:1696-1719.

92. Waanders LF, Chwalek K, Monetti M, Kumar C, Lammert E, Mann M. Quantitative proteomic analysis of single pancreatic islets. Proc Natl Acad Sci U S A 2009; 106:18902-7.

93. Behringer R, Gertsenstein M, Vintersen Nagy K, Nagy A. Manipulating the Mouse Embryo: A Laboratory Manual, Fourth Edition. Cold Harb Lab Press 2014.

94. Kang YJ, Forbes K, Carver J, Aplin JD. The role of the osteopontin-integrin av $\beta 3$ interaction at implantation: Functional analysis using three different in vitro models. Hum Reprod 2014; 29:739749.

95. Kong $C$, Sun L, Zhang M, et al. miR-133b reverses the hydrosalpinx-induced impairment of embryo attachment through down-regulation of SGK1. J Clin Endocrinol Metab 2016; 101:1478-1489.

96. Gu X-W, Chen Z-C, Yang Z-S, et al. Blastocyst-induced ATP release from luminal epithelial cells initiates decidualization through the P2Y2 receptor in mice. Sci Signal 2020; 13:eaba3396.

97. Benjamini Y, Hochberg Y. Controlling the False Discovery Rate: A Practical and Powerful Approach to Multiple Testing. J R Stat Soc Ser B 1995; 57:289-300.

98. Szklarczyk D, Gable AL, Lyon D, et al. STRING v11: protein-protein association networks with increased coverage, supporting functional discovery in genome-wide experimental datasets. Nucleic Acids Res 2019; 47:D607-D613.

99. Huang DW, Sherman BT, Tan Q, et al. DAVID Bioinformatics Resources: expanded annotation database and novel algorithms to better extract biology from large gene lists. Nucleic Acids Res 2007; 35:W169-W175.

100. Huang DW, Sherman BT, Lempicki RA. Systematic and integrative analysis of large gene lists using DAVID bioinformatics resources. Nat Protoc 2009; 4:44-57.

101. Wickham H. ggplot2: Elegant Graphics for Data Analysis. Springer-Verlag New York; 2016.

102. Subramanian A, Tamayo P, Mootha VK, et al. Gene set enrichment analysis: A knowledge-based approach for interpreting genome-wide expression profiles. Proc Natl Acad Sci 2005; 102:1554515550.

103. Mootha VK, Lindgren CM, Eriksson K-F, et al. PGC-1 a-responsive genes involved in oxidative phosphorylation are coordinately downregulated in human diabetes. Nat Genet 2003; 34:267-273.

104. Genome Informatics ... 2009; 5:2-3.

105. Desta IT, Porter KA, Xia B, Kozakov D, Vajda S. Performance and Its Limits in Rigid Body ProteinProtein Docking. Structure 2020; 28:1071-1081.e3. 
106. Vajda S, Yueh C, Beglov D, et al. New additions to the ClusPro server motivated by CAPRI. Proteins Struct Funct Bioinforma 2017; 85:435-444.

107. Kozakov D, Hall DR, Xia B, et al. The ClusPro web server for protein-protein docking. Nat Protoc $2017 ; 12: 255-278$.

108. Kozakov D, Beglov D, Bohnuud T, et al. How good is automated protein docking? Proteins Struct Funct Bioinforma 2013; 81:2159-2166.

Figures 
A
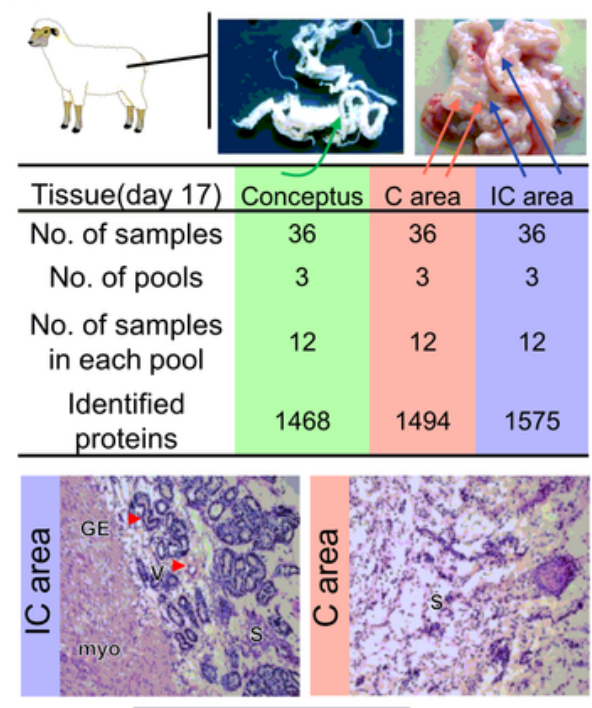

B

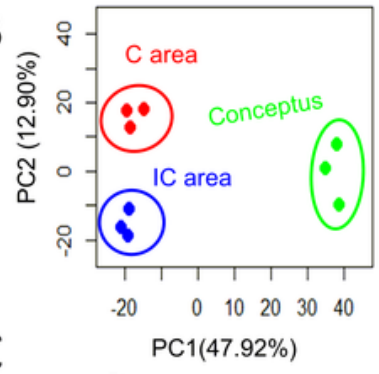

C
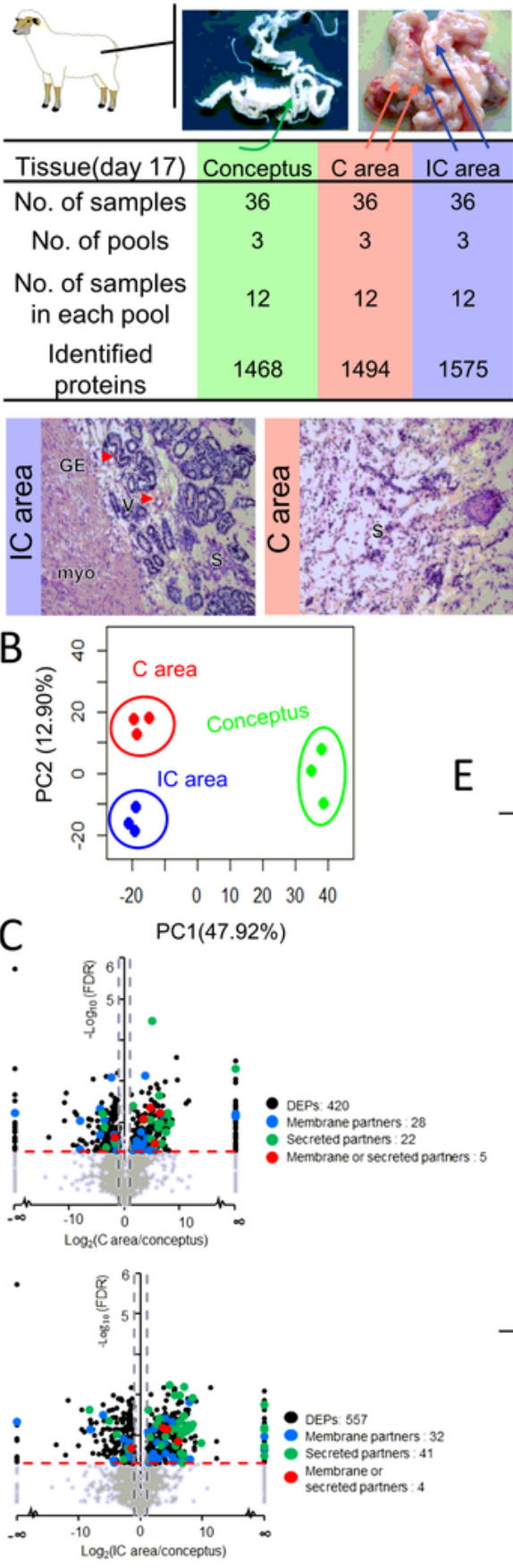

$\mathrm{E}$
D
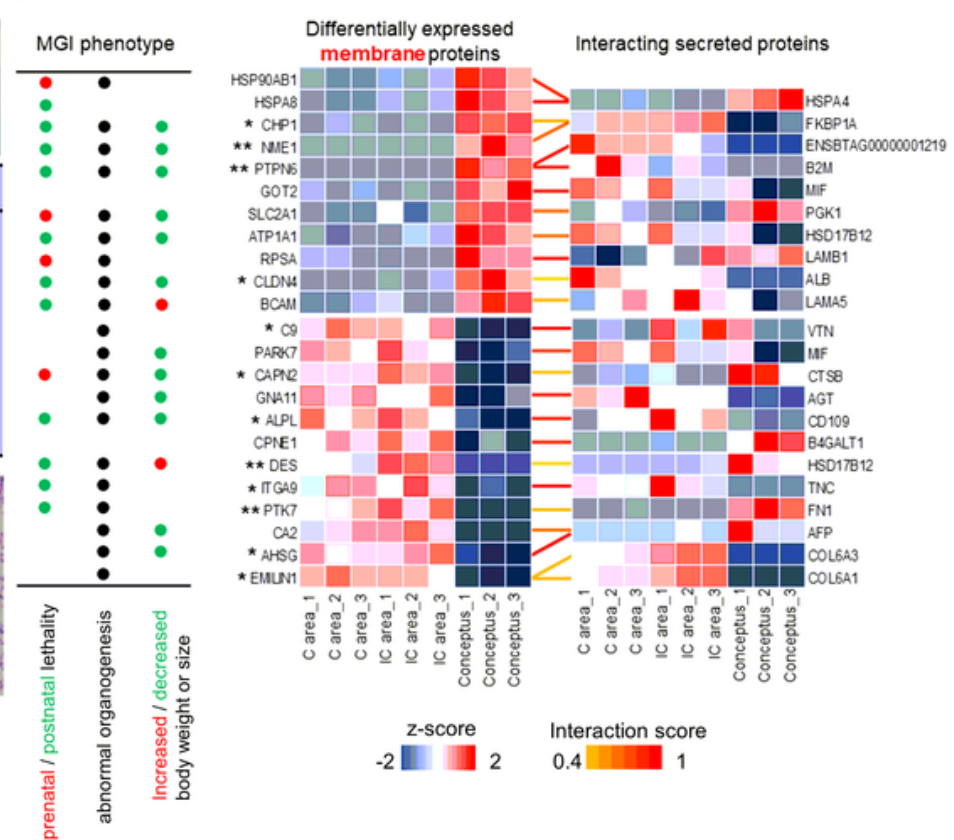

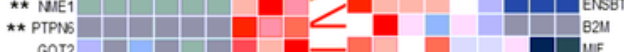

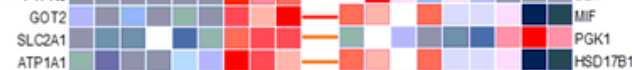

ACPA1
RPSA
RPSA

* CLDNA
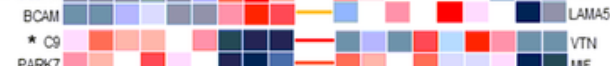

PARK77
$*$ CAPNR

* GNA11

^ALPL

CPIE1

$\star \star \star D E S$
$\star \pi G A 9$

$\star \pi G A 9$
$\star \star P T K 7$

*A2

* EMLSW

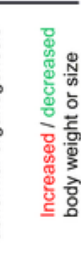

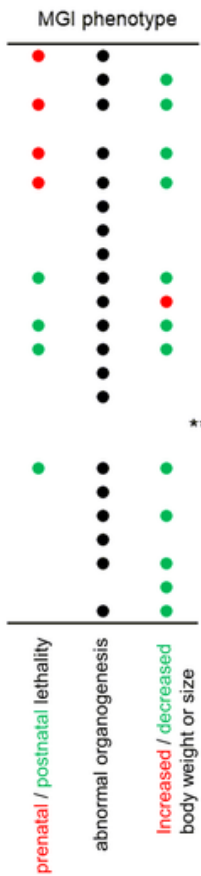

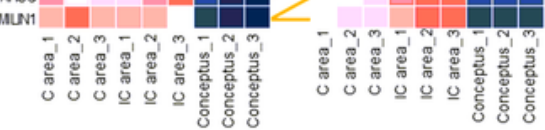

z-score

\begin{tabular}{lll}
-2 & Interaction score \\
\hline & & 0.4
\end{tabular}

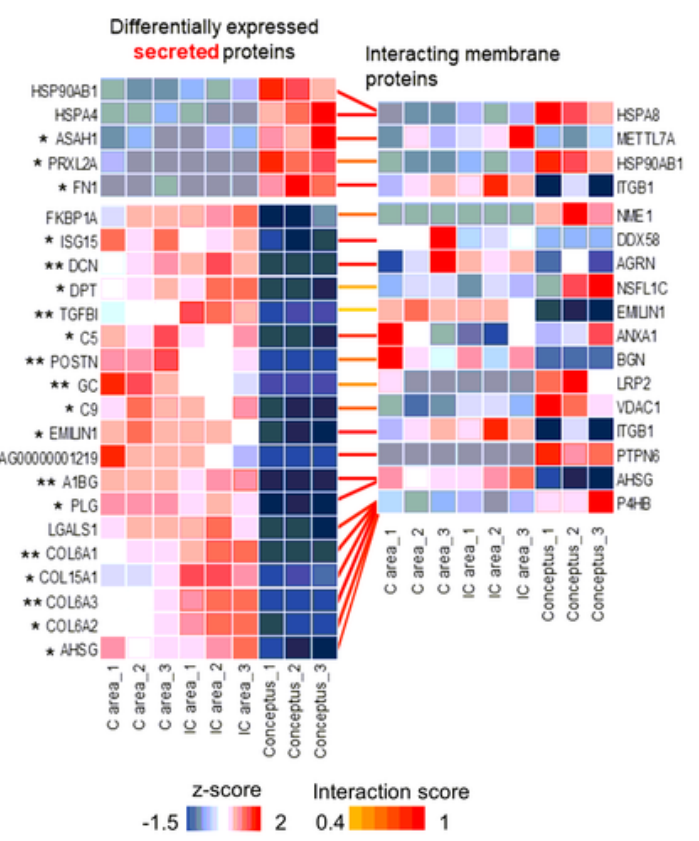

\section{Figure 1}

Screening of differentially abundant membrane or secreted proteins and their interacting partners between the conceptus and endometrium. (A) Data structure used in this study. Representative images of a pregnant uterus and conceptus. Data of genome-wide protein abundance was obtained from the conceptus, as well as the $\mathrm{C}$ and IC areas from thirty-six pregnant uteri, which were equally divided into three pools, twelve samples in each pool. Right panel: Representative hematoxylin-eosin staining images 
of $C$ and IC areas identifying the tissues from which proteomic data were used in this study. GE, glandular epithelium; S, stroma; myo, myometrium; V, blood vessels. (B) Principal component analysis of protein expression patterns of three biological replicates of the conceptus, C area, and IC area. (C) Volcano plot of differentially abundant proteins between the conceptus and the $\mathrm{C}$ area (upper panel) or IC area (lower panel). The black dots represent the DAPs with FDR $<0.05$ and FC $>2$. The red line represents FDR $=0.05$, and the gray line represents $F C=2$. The blue dots represent identified membrane partners. The green dots represent identified secretion partners. The red dots represent proteins that could be secreted or located in membrane. (D) and (E) Overview of the interactions of membrane/secreted proteins that were significantly changed in both $\mathrm{C}$ and IC areas compared with that in the conceptus, with their interacting secreted/membrane partners. The Z-score normalized protein abundance is represented in red (relatively high) or blue (relatively low). * represents the fold change of the protein in the conceptus versus that in the $\mathrm{C}$ areas and in the conceptus versus that in the $\mathrm{IC}$ areas, both $>10$. ** represents the fold change $>$ 100. The interaction scores are represented in yellow (0.4) to red (1). Left panel: The representative Mouse Genome Informatics (MGI) phenotypic annotations of differentially abundant membrane proteins. 


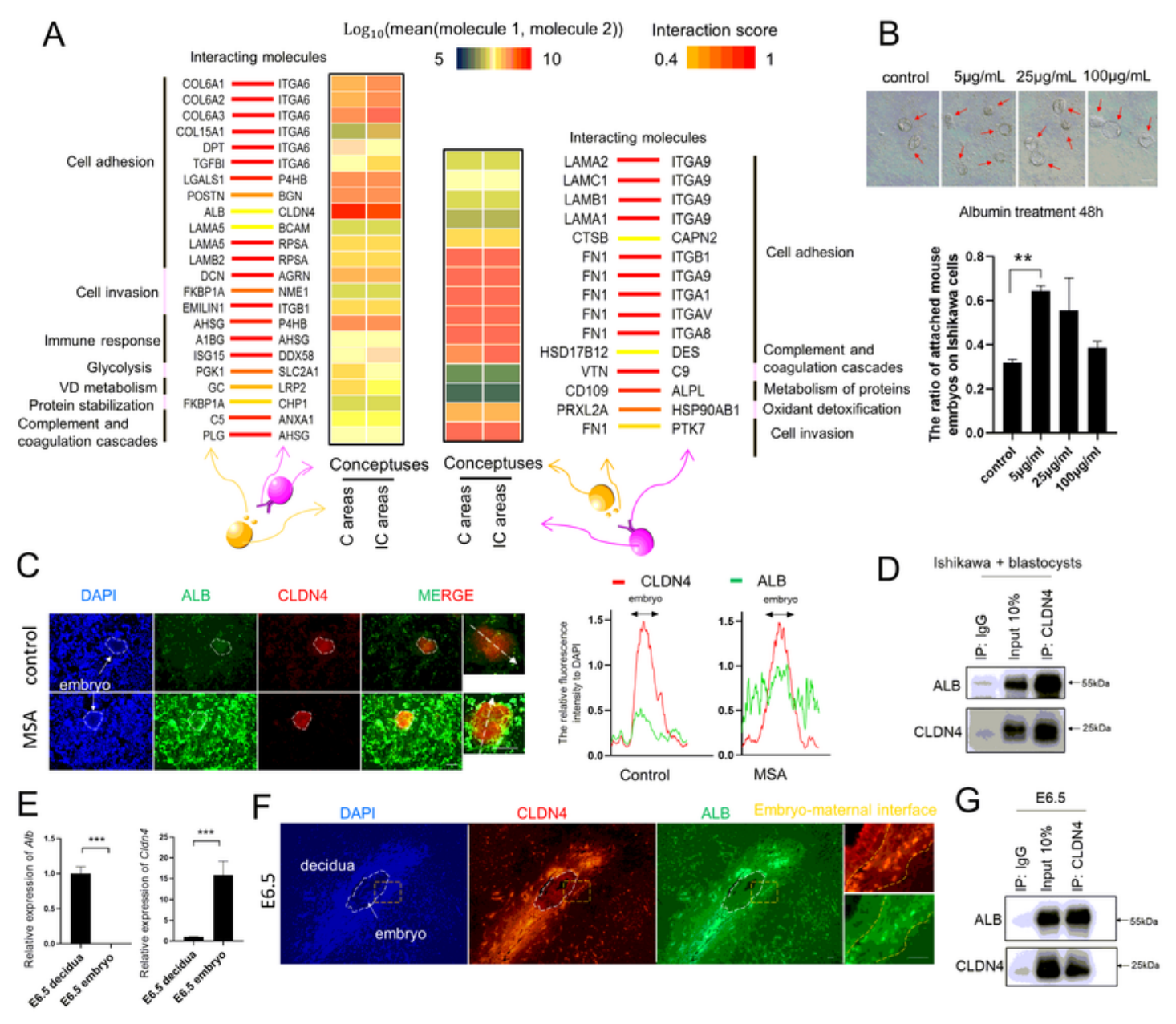

\section{Figure 2}

Working model of secreted-membrane partner interactions and related essential biological processes that govern crosstalk at the maternal-fetal interface. (A) Overview of selected secreted-membrane partner interactions-related biological processes. The interaction scores are represented in yellow (0.4) to red (1). The average expression level of interacting molecule 1 in cluster 1 and interacting molecule 2 in cluster 2 are indicated by colors (blue to red). (B) The attachment of mouse blastocysts to Ishikawa cells was 
assayed after 48 hours of coculture supplemented with different concentrations of albumin from bovine serum. Representative images are shown and the red arrows represent the firmly attached embryos. ** represents $P<0.01$. (C) The co-localization of ALB and CLDN4. Left panel: Representative fluorescent images of ALB (green), CLDN4 (red), and DAPI (blue) staining in mouse embryos co-cultured 48 hours with Ishikawa cells. Control and MSA group were incubated in FBS-medium and MS-medium 30 min before fixed, separately. Right panel: Intensity profiles of ALB and CLDN4 relative to DAPI obtained using ImageJ software, along the white dotted straight line crossing Ishikawa cells and blastomeres. White arrows represent the attached embryos. (D) Western blot showing co-immunoprecipitated proteins in MSA group of Ishikawa cells co-cultured with blastocysts immunoprecipitation experiments, which were repeated at least twice with similar results. (E) Quantification of Alb and Cldn4 mRNAs relative to Actb in the E6.5 decidua and embryo. (F) Representative fluorescent immunohistochemistry images of DAPI (blue), ALB (green), and CLDN4 (red) staining in E6.5. The white dotted circles represent the embryo sites. The areas between two yellow dotted lines represent maternal-fetal interface. (G) Western blot showing co-immunoprecipitated proteins in E6.5 immunoprecipitation experiments, which were repeated at least twice with similar results. Scaled bar $=200 \mu \mathrm{m}(\mathrm{B}, \mathrm{C}$, and F). 


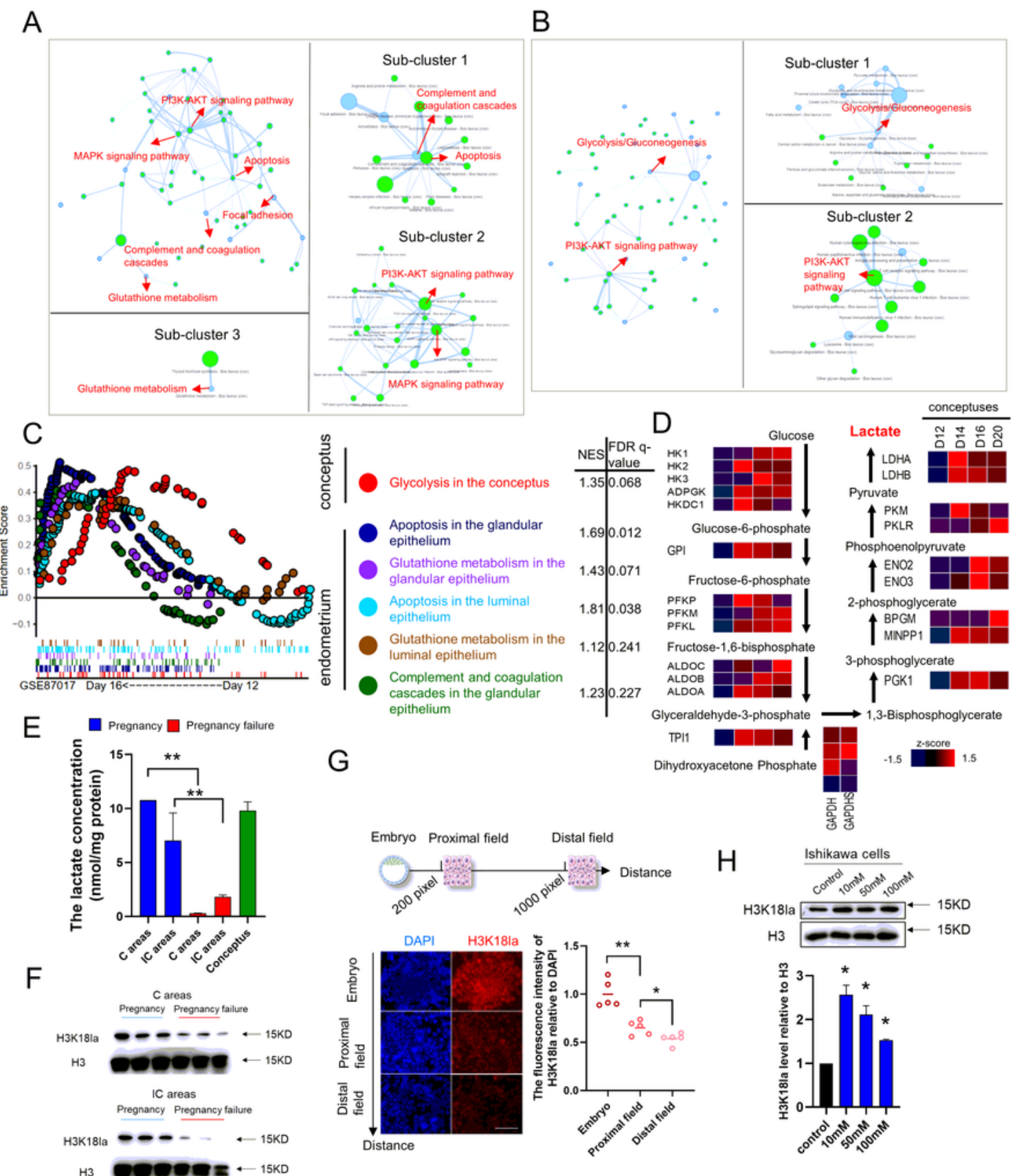

\section{Figure 3}

The key pathways and processes at the maternal-fetal interface. (A) The pathway network enriched in the endometrium was constructed by PathwayConnector (PC). The key pathways are marked with red arrows, and the important sub-clusters are presented separately. The blue dots represent the input pathways, and the green dots represent the complementary pathways. (B) The pathway network enriched in the conceptus was constructed using PathwayConnector (PC). (C) Differentially regulated pathways between 
day 16 and day 12 conceptuses, the glandular epithelium, and the luminal epithelium were identified by GSEA (NES > 1, FDR q-value < 0.25). (D) Dynamic expression of glycolytic enzymes in the conceptus from day 12 to day 20 of pregnancy (GSE87017). The Z-score normalized RPKM is represented in red (relatively high) and blue (relatively low). (E) Lactate concentrations of the sheep conceptus and endometrial tissues with successful and failed pregnancy by implantation stage. ** represents $P<0.01$. (F) Immunoblots of H3K18la and H3 from the endometrium with successful and failed pregnancy by implantation stage. (G) Representative fluorescent images of H3K18la (red) and DAPI (blue) staining in mouse embryos co-cultured 72 hours with Ishikawa cells. The intensity profiles of H3K18la relative to DAPI of Ishikawa cells obtained using ImageJ software, which was stronger at proximal fields than that at distal fields. * represents $P<0.05$, ** represents $P<0.01$. Scale bar $=200 \mu \mathrm{m}$. $(\mathrm{H})$ Immunoblots of $\mathrm{H} 3 \mathrm{~K} 18 \mathrm{la}$ and $\mathrm{H} 3$ in Ishikawa cells exposure to different concentrations of sodium L-lactate. Lower panel: The relative intensity of H3K18la. * represents $\mathrm{P}<0.05$ relative to the control group. 
A

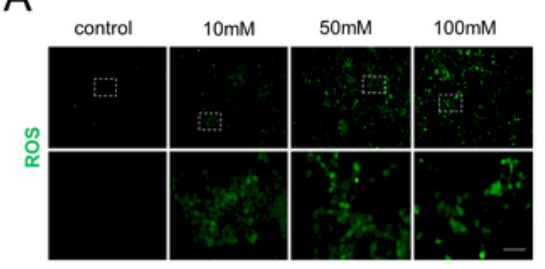

B

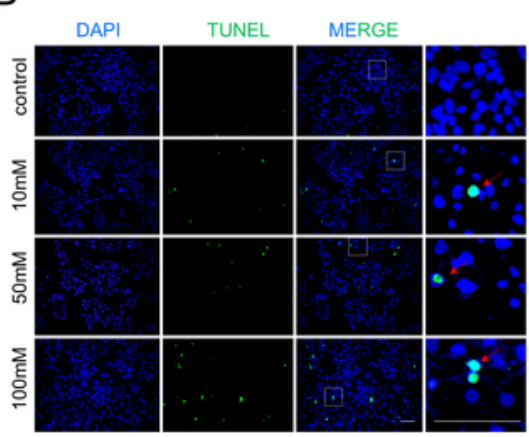

F

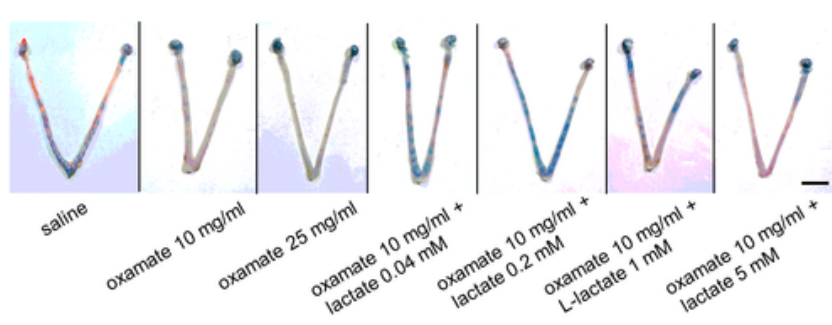

C
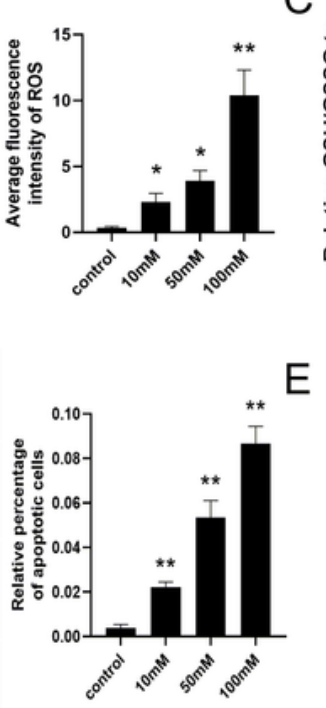
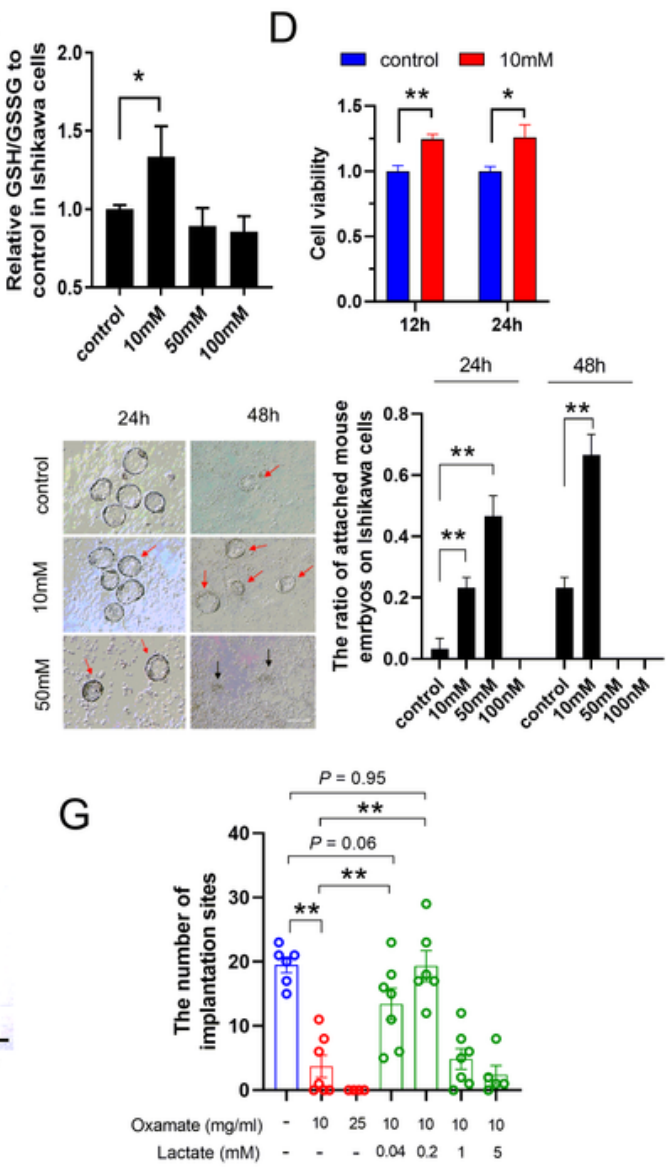

$\mathrm{H}$

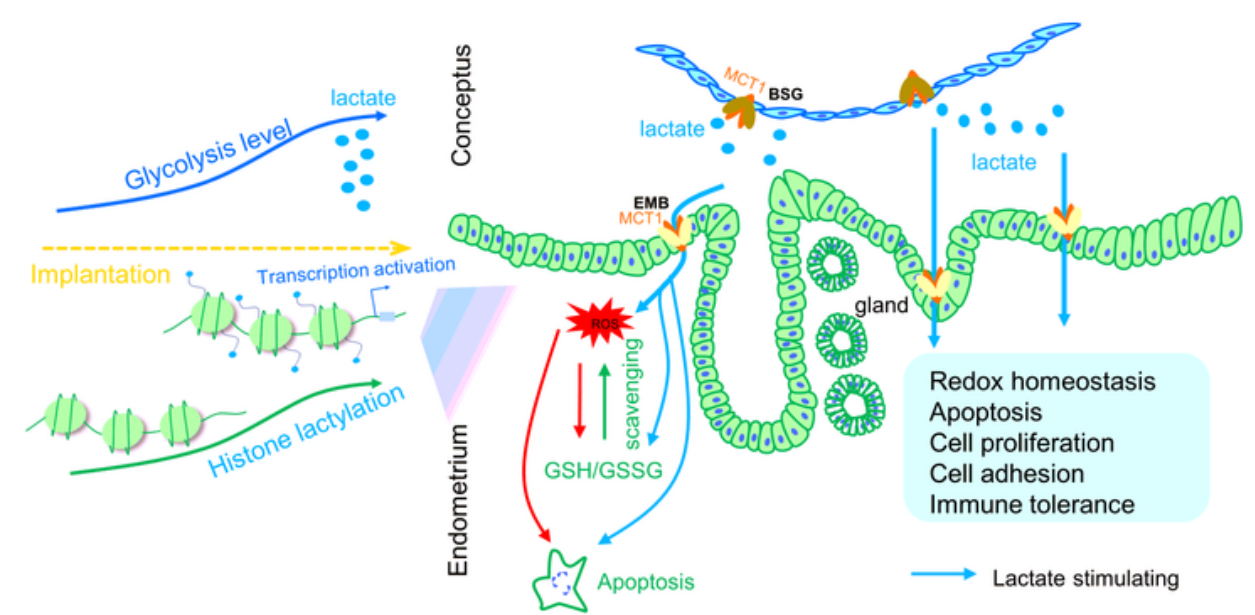

\section{Figure 4}

The adaptive response of the endometrium to the embryonic lactate signal. (A) Representative fluorescent images of ROS production (green) in human endometrial cancer cells (Ishikawa line) exposed to different concentrations of sodium L-lactate. Right panel: Normalized fluorescent intensity of ROS. * represents $P<0.05$, ** represents $P<0.01$ relative to control group. (B) Representative fluorescent images of TUNEL (green) and DAPI (blue) staining in Ishikawa cells exposed to different concentrations of 
sodium L-lactate. Right panel: Quantification of TUNEL-positive Ishikawa cells following different treatments. ** represents $P<0.01$ relative to the control group. (C) The GSH/GSSG ratio in different sodium L-lactate concentrations treated Ishikawa cells was measured. * represents $P<0.05$. (D) Proliferation assay of Ishikawa cells exposed to $10 \mathrm{mM}$ sodium L-lactate for $12 \mathrm{~h}$ and $24 \mathrm{~h}$. ** represents $P<0.01$, * represents $P<0.05$. (E) The attachment of mouse blastocysts to Ishikawa cells was assayed after 24 and 48 hours of coculture supplemented with different concentrations of sodium L-lactate. Representative images are shown and the red arrows represent the firmly attached embryos, and the black arrows represent the degraded embryos. ${ }^{*}$ represents $P<0.01$. ( $F$ and $\left.G\right)$ Uterine morphology $(F)$ and the number of implantation sites $(G)$ on day 5 after uterine horns at day 4 were injected with different treatments. ${ }^{* *}$ represents $P<0.01$. Scale bar $=200 \mu \mathrm{m}(A, B$, and E). Scale bar $=1 \mathrm{~cm}(F)$. (H) Schematic diagram of the endometrial response to embryonic lactate signals during implantation. Glycolytic metabolism is upregulated in the conceptus, thus the conceptus-derived lactate would serve as an embryonic signal to promote histone lactylation in the endometrium, which in turn participates in the regulation of redox homeostasis, apoptosis, cell proliferation, cell adhesion, and immune tolerance. Blue arrows represent lactate stimulation, red arrows represent ROS stimulation, and the green arrow represents ROS scavenging by GSH/GSSG homeostasis.

\section{Supplementary Files}

This is a list of supplementary files associated with this preprint. Click to download.

- CBSI.docx

- supplementarytable1.xlsx

- supplementarytable2.xlsx

- supplementarytable3.xlsx 